\title{
红紫素-18 的化学修饰及其叶绿素类二氢卟吩衍生物的合成
}

\author{
刘红瑶 $a$ 朱国华 ${ }^{a}$ 刘由再 ${ }^{b}$ 金英学 $*, a$ 祁彩霞 $c$ 王进军*, $b, c$ \\ ( ${ }^{a}$ 哈尔滨师范大学化学化工学院 哈尔滨 150025)

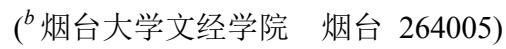 \\ $\left({ }^{c}\right.$ 山东省黄金工程技术研究中心(工业应用) 烟台 264005)
}

\begin{abstract}
摘要 以红紫素-18 甲酯及其 $N$-甲氧基二酰亚胺甲酯为起始原料, 以氧化苯甲腈或者甲亚胺作为 1,3 -偶极体, 与红紫 素-18 的 3-位乙烯基进行偶极环加成反应, 在周环上建立了不同的五元杂环结构; 选择芳胺和芳醛缩合而成的含氮二烯 与红紫素-18 二酰亚胺 C(3)-双键实施杂 Diels-Alder 反应(Povarov 反应), 得到了 3-位芳酰基或者芳烷基取代的开环重排 产物; 利用 20-meso-氢和 C(12)-甲基的反应活性，在红紫素-18 的二氢卟吩色基上分别完成了亲电取代、空气氧化和类 羟醮缩合反应, 并在周环的 12-和 20-位上引进了不同的取代基团, 合成了一系列未见报道的叶绿素类二氢卟吩衍生物. 其化学结构均经 UV, IR, ${ }^{1} \mathrm{H}$ NMR, MS 及元素分析予以证实. 同时, 对所涉及的反应机理也进行了相应的讨论.
\end{abstract}

关键词 叶绿素-a; 二氢卟吩; 红紫素-18; 化学反应; 合成

\section{Chemical Modifications of Purpurin-18 and Synthesis of Chlorophyllous Chlorins Derivatives}

\author{
Liu, Hongyao ${ }^{a}$ \\ Zhu, Guohua ${ }^{a}$ \\ Liu, Ranran ${ }^{b} \quad$ Jin, Yingxue ${ }^{*, a}$ \\ Qi, Caixia ${ }^{c}$ \\ Wang, Jinjun ${ }^{*, b, c}$ \\ ( ${ }^{a}$ College of Chemistry \& Chemical Engineering, Harbin Normal University, Harbin 150025) \\ ( ${ }^{b}$ Wenjing College, Yantai University, Yantai 264005) \\ ( ${ }^{c}$ Shandong Applied Research Centre of Gold Nanotechnology (Au-SDARC), Yantai 264005)
}

\begin{abstract}
Purpurin-18 methyl ester and its imid ester were used as starting materials. The cycloaddition of the 3-vinyl group in purpurin-18 with nitril oxide or azomethine as 1,3-dipole was carried up to established different five-membered heterocyclic structures on the chlorin chromophore. Azadiene, prepared from the condensation of arylamine with aromatic aldehyde, with double bond at 3-position of purpurin-18 conducted hetero-Diels-Alder reaction (Povarov reaction) to generate ring-opening rearranged products substituted with aroyl or aralkyl group. The electrophilic substitution, allomerization and like-Aldol reaction were accomplished making use of the reactivity of $\mathrm{C}(12)$-methyl or 20-meso-hydrogen to introduce various substitutes at 12- and 20-position on the periphery of purpurin-18. The synthesis of a series of unreported chlorins related to chlorophyll was accomplished and their chemical structures were characterized by elemental analysis, UV, IR and ${ }^{1} \mathrm{H}$ NMR spectra. The reaction mechanisms involved were also discussed accordingly.

Keywords chlorophyll-a; chlorin; purpurin-18; chemical reaction; synthesis
\end{abstract}

红紫素-18 是叶绿素-a 多阶降解过程中所形成的一 个重要的中间产物, 其多取代的芳香性二氢卟吩母环和 六元环酐外接环结构形成了独特的活性反应区域, 同时 也显示出优良的光物理和光化学性质 ${ }^{[1,2]}$. 红紫素-18 可 以顺利地转换成二氢卟吩- $e_{6}$ 、二氢卟吩- $p_{6}$ 、红紫素-7、 罗多二氢卟吩等多种具有叶绿素-a 基本环系的二氢卟 吩衍生物; 作为新一代光动力肿瘤治疗 (Photodynamic Therapy, PDT)药物的理想前体, 红紫素-18 为合成高效 低毒的 PDT 药物(光敏剂)建立了有效的切入途径 ${ }^{[3]}$. 因 此, 探讨红紫素-18 及其衍生物的化学行为, 改善和优

* E-mail: wjj1955@163.com

Received October 6, 2014; revised December 22, 2014; published online January 9, 2015

Project supported by the National Natural Science Foundations of China (No. 21272048) and the Project of Shandong Applied Reaearch Centre of Gold Nanotechnology (2011).

国家自然科学基金(No. 21272048)和山东省黄金工程技术研究中心(2011 年度)资助项目. 
化四吡咯大环分子的理化性质, 有益于拓展叶绿素的化 学研究及其二氢卟吩衍生物的实际应用. 红紫素-18 的 结构修饰主要是集中于外接环酐的胺解反应和 3-位乙 烯基的氧化还原反应 ${ }^{[4]}$. 本文以红紫素-18 甲酯(1)为起 始原料, 通过 1,3-偶极环加成、亲电取代、空气氧化和 类羟醛缩合等反应对其周环结构的化学修饰, 完成了一 系列具有红紫素-18 基本碳架的叶绿素类二氢卟吩衍生 物的合成, 为笁选新型光动力肿瘤治疗药物提供了具有 应用前景的待选四吡咯大环化合物.

在碱性条件下, 以芳甲醛肟与 NBS 所形成的氧化 苯甲腈与红紫素-18 (1a)发生 1,3-偶极环加成反应, 均得 到中等产率的 C(3)-异噁唑啉基取代的红紫素-18 衍生物 $(\mathbf{2} \mathbf{a} \sim 2 \mathbf{c})$, 同时, 每次反应都分离出少量的 3-甲酰基红 紫素-18 (3); 选择 4-氯靛红在碱性条件下与肌氨酸缩合, 所得甲亚胺类 1,3-偶极体与起始原料 $1 \mathbf{a}$ 进行相同的偶 极环加成反应, 分离出两个产率相当的连有氮杂螺环结 构的红紫素-18 衍生物 $4 \mathbf{a}$ 和 $\mathbf{4 b}$. 参考 Povarov 反应的环 合机理 ${ }^{[5]}$, 在三氟化嗍的催化下, 将 $1 \mathbf{b}$ 、芳胺和芳醛混 合在一起于甲苯中共回流，尝试采用一锅法完成相应的
杂-Diels-Alder 反应，其反应结果并未给出预期产物，分 别得到了 3-位芳酰和芳烷基取代的紫红卟啉二酰亚胺 $5,(5 \%), 6(7 \%)$ 以及红紫素-18 二酰亚胺 7 (25\%).

红紫素-18 (1a)在吡啶中与氨甲基吡啶的缩合反应 也以理想的产率给出 $N$-吡啶甲基红紫素-18 二酰亚胺 $(\mathbf{8}$, 69\%); 在氢氧化锂促进下, 红紫素-18 二酰亚胺 $1 \mathbf{b}$ 与空 气中单线态氧发生反应，12-位甲基氧化成甲酰基而转 化成 $31 \%$ 的二氢卟吩醛(9); 在碱性条件下, 利用 12-位 甲基的类 $\alpha$-位氢的反应活性，与苯甲醛进行羟醛缩合反 应，以 $43 \%$ 的产率分离出 12-羟苯乙基取代的红紫素-18 二酰亚胺 $(\mathbf{1 0})$, 然后在对甲苯磺酸的催化下, 于二氯乙 烷溶液中室温脱水形成 12-苯甲叉基二酰亚胺(11, 65\%). 在乙酸存在下，选择发烟硝酸为硝化剂对二酰亚胺 $\mathbf{1 b}$ 实施亲电取代反应，选择性地在 20-位上引进了硝基， 并以 38\%的产率得到 20-硝基红紫素-18 衍生物 12; 以 $N$-氯代琥珀酰亚胺 $(\mathrm{NCS})$ 与二氢卟吩醛 9 进行氯代反应, 同样在 20-位上引进了新的取代基团，以 49\%的收率获 得 20-氯-12-甲酰基红紫素-18 二酰亚胺(13).

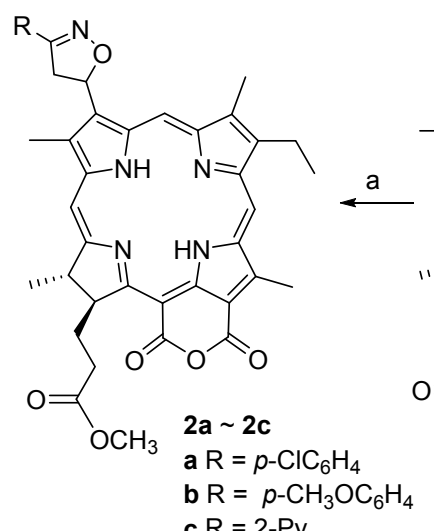
c $\mathrm{R}=2-\mathrm{Py}$

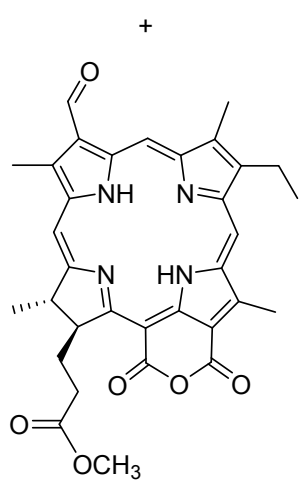

3

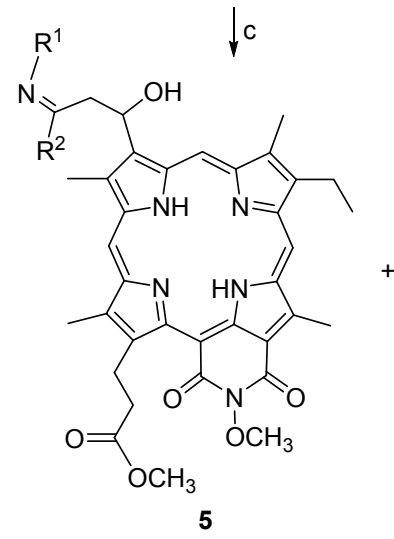

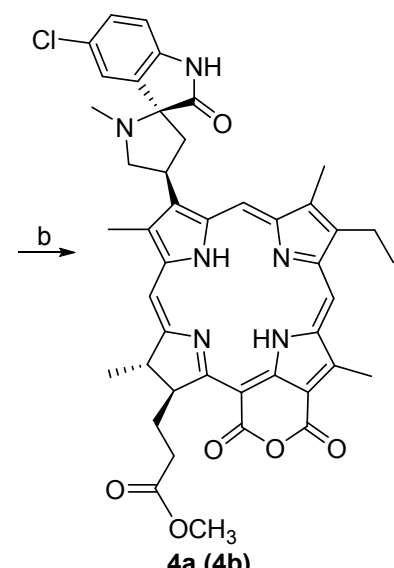
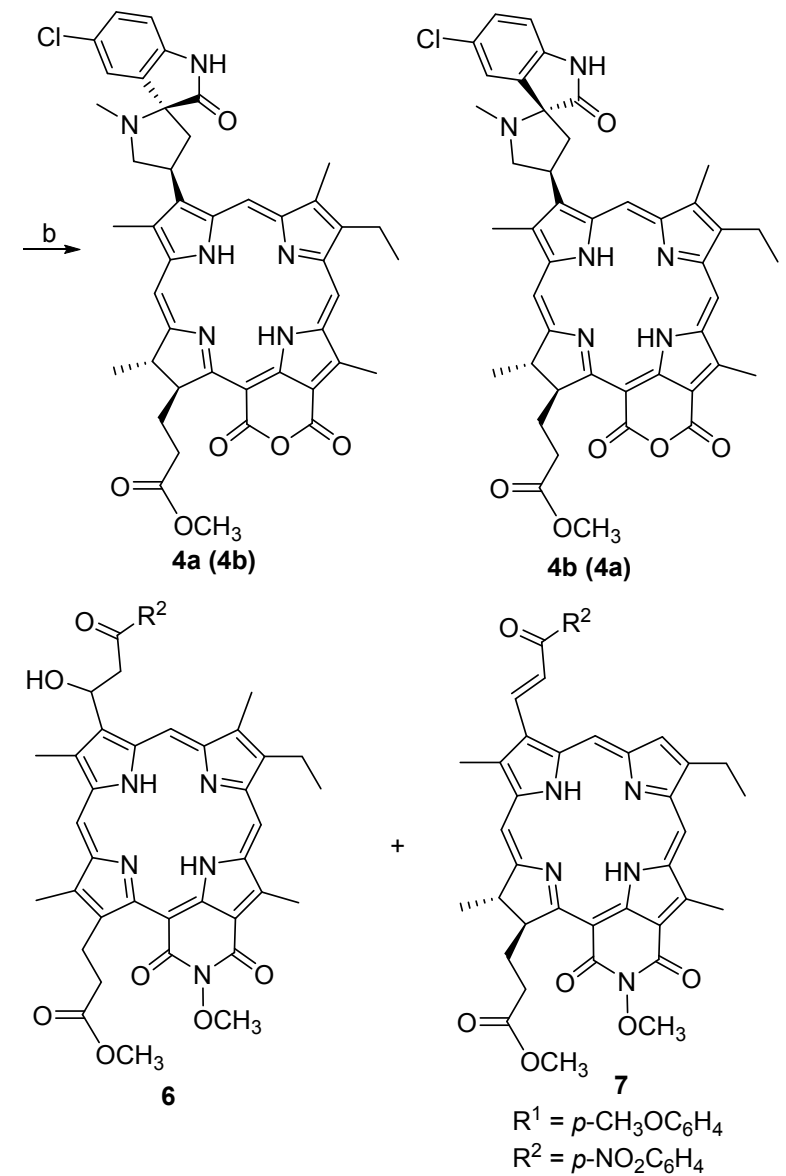

Reagents and conditions: (a) RNOH/TEA/NBS; (b) 4-Cl-isatin/ $\mathrm{CH}_{3} \mathrm{NCH}_{2} \mathrm{CO}_{2} \mathrm{H} /$ Toluene; (c) $p-\mathrm{CH}_{3} \mathrm{OC}_{6} \mathrm{H}_{4} \mathrm{NH}_{2} / p-\mathrm{NO}_{2} \mathrm{C}_{6} \mathrm{H}_{4} \mathrm{CHO}_{3} / \mathrm{BF}_{3}, \mathrm{Et}_{2} \mathrm{O} / \mathrm{Toluene}$

图式 1 红紫素-18 甲酯 3-位乙烯基的环加成反应

Scheme 1 The cycloaddition of the vinyl group at 3-position in purpurin-18 eater 


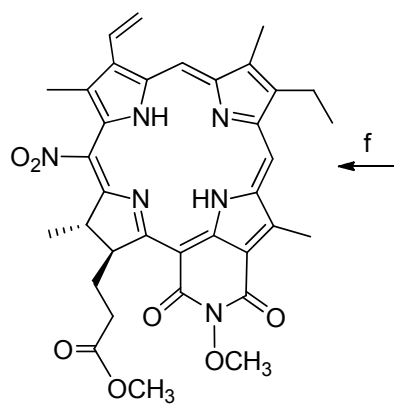

12

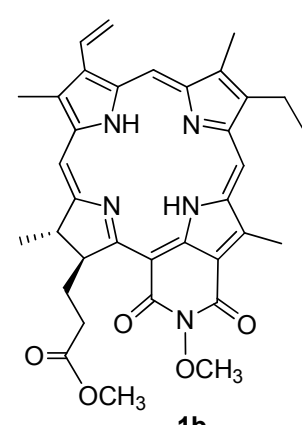

1b

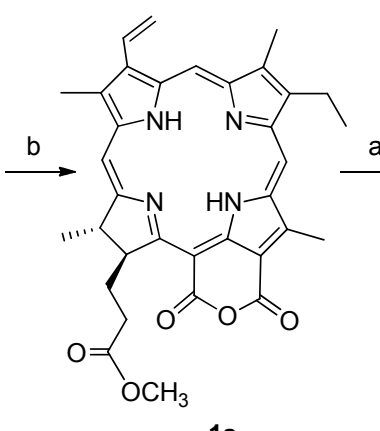

1a

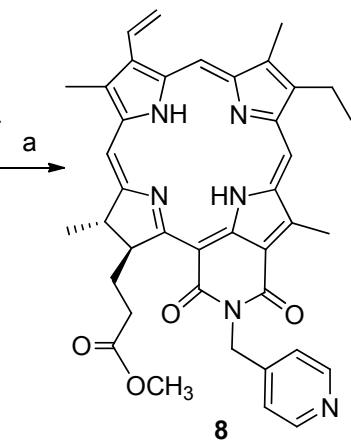

8

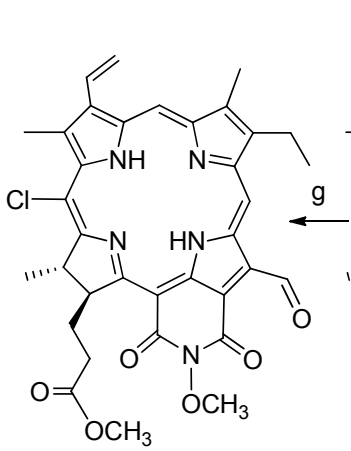

13

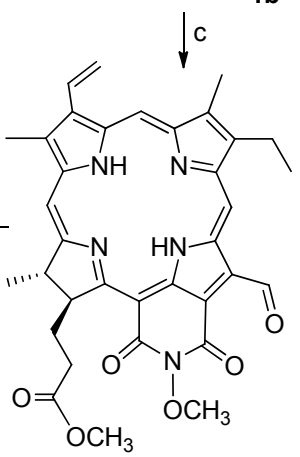

9

$\searrow^{d}$

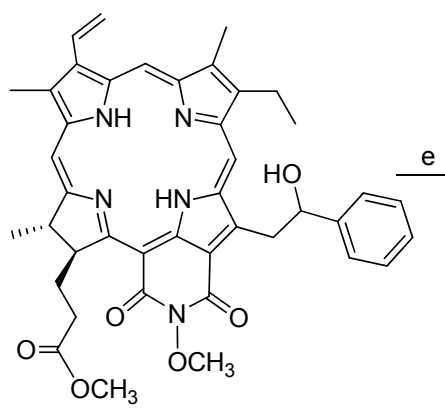

10

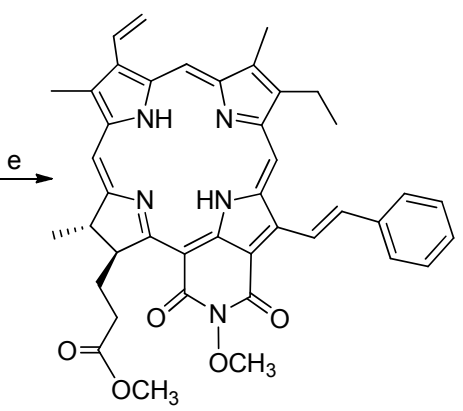

11

Reagents and conditions: (a) $\mathrm{PyCH}_{2} \mathrm{NH}_{2} / \mathrm{Pyr}$; (b) $\mathrm{NH}_{2} \mathrm{OH} \cdot \mathrm{HCl} / \mathrm{Pyr}$; (c) $\mathrm{LiOH} / \mathrm{THF} / \mathrm{MeOH}$; (d) $\mathrm{NaOMe} / \mathrm{MeOH} / \mathrm{PhCHO}$; (e) TsOH/CH $\mathrm{Cl}_{2}$; (f) $\mathrm{AcOH} / \mathrm{HNO}_{3} ;$ (g) $\mathrm{NCS} / \mathrm{MeOH} / \mathrm{CH}_{2} \mathrm{Cl}_{2}$

图式 2 红紫素-18 二酰亚胺周环上的化学反应

Scheme 2 The reactions along the perphery of purpurin-18 imide

\section{1 结果与讨论}

\section{1 红紫素-18 及其二酰亚胺中 3-位乙烯基的环合反} 应

与其他叶绿素降解产物一样, 作为活性反应区域, 红紫素-18 的 3-位、12-位和 20-meso-位同样可以发生多 种典型的化学反应, 特别是 3-位乙烯基的 1,3-偶极环加 成反应, 为在二氢卟吩周环上构建新型杂环取代结构提 供了一个非常重要的合成方法. 由于 3-位乙烯基与红紫 素-18 的芳香性氮杂轮烯色基相连, 因此, 与苯乙烯环 上双键的电荷分布相似，在亲偶极性双烯的 3a-和 3b-位 上的组合系数不同, 端烯碳原子上的组合系数相对更 大; 另一方面, 在 1,3-偶极体的最低未占轨道(LUMO) 中碳原子拥有最大的组合系数, 而在最高占有轨道 $(\mathrm{HOMO})$ 中, 最大组合系数则落到端位杂原子上 ${ }^{[6]}$. 因 此, 偶极体的 LUMO 轨道和亲偶极体的 HOMO 轨道在 环合过程中优先发生作用, 即生成五元异噁唑啉基取代 的二氢卟吩 $\mathbf{B}$ 而不是生成 A. 靛红在与红紫素-18 进行 环化时同样表现出良好的区域选择性, 首先, 靛红与肌 氨酸相互作用形成亚胺盐 $\mathbf{a}$, 在碱性条件下脱去二氧化 碳给出 1,2-偶极体 $\mathbf{b}$, 其共振杂化体 $\mathbf{c}$ 则是参与环加成 反应的 1,3-偶极体. 由于甲亚胺偶极体的两端均为碳原 子, 因此, 在最低未占轨道(LUMO) 和最高占有轨道
(HOMO)中端位原子的组合系数相似, 而对环合的取向 起决定性作用的则是 1,3-偶极体自身的立体结构, 即带 有吲哚酮结构一侧的偶极远离二氢卟吩色基而连接在
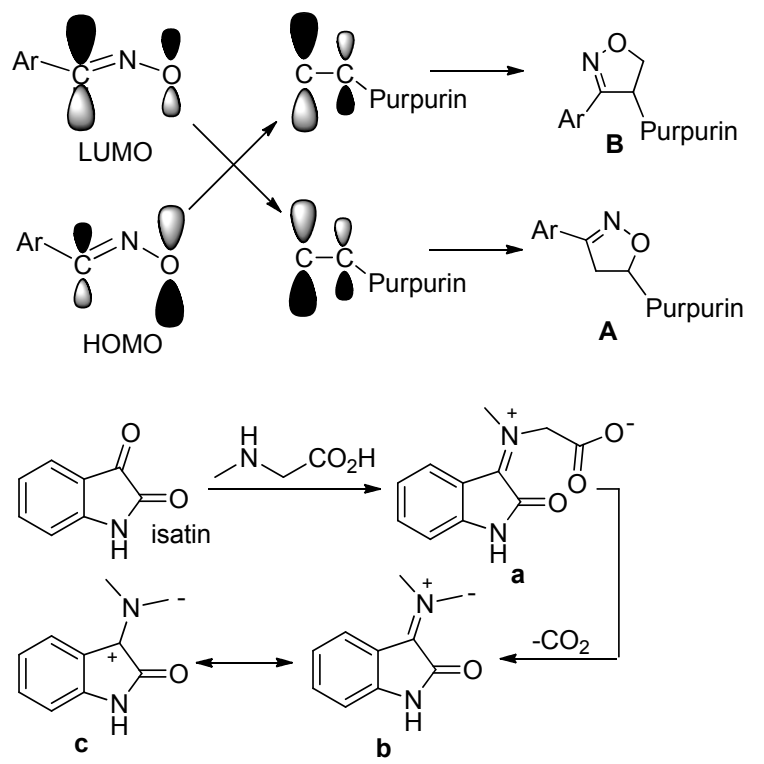

图 1 红紫素-18 与氧化苯甲腈或甲亚胺在 1,3-偶极环合反应 中的区域选择性

Figure 1 The regioselectivity in 1,3-dipolar cycloaddition of purpurin-18 with nitril oxide or azomethine 
3b-碳原子上，以规避两个环系之间形成更大的空间位 阻.

除了区域选择性以外, 红紫素-18 或者其二酰亚胺 与 1,3-偶极环体的环加成反应也体现出相应的立体选择 性. 由于红紫素-18 的氢化吡咯环(D-子环)中两个 $\mathrm{sp}^{3}$ 杂 化的 $\beta$-碳原子均具有手性, 因而缺失环结构共平面的基 本元素, 上下两面的化学环境也存在着一定程度的区 别. 对于 3-位烯键而言, 1,3-偶极体从上下两面都可以完 成环合作用, 形成过渡态所需要的活化能也有所不同, 因此偶极体对环上碳碳双键的反应取向则有所选择. 红 紫素-18 与 1,3-偶极体的环加成都没有给出等同的差向 异构体, 与氧化苯甲腈和靛红甲亚胺的环加成产物中 $R / S$ (或者 $S / R$ )构型的产物比例都大约为 $8: 7$. 由于吲哚 酮亚胺偶极体占有较大的空间, 所生成的差向异构体之 间存在着明显的立体差异, 因此, 分离出两个难以确定 构型并且具有阻旋异构特征的立体异构体.

根据杂-Diels-Alder 反应(Povarov 反应)的作用机理 和叶绿素类二氢卟吩色基的立体结构特性 ${ }^{[7]}$, 芳胺和芳 醛缩合而成的亚胺具有氮二烯结构, 与 $1 \mathrm{~b}$ 发生环合后, 可以形成两个非对映异构体 A1 和 A2; 如果继续沿循 Povarov 反应的步骤发展, 则要通过碱的作用异构化, 进而恢复芳环体系给出四氢喹啉取代的二氢卟吩 $\mathbf{B}$. 由 于在 3-位上形成氢化喹啉环状结构将导致很大的空间 位阻, 因而在苯胺与三氟化硼共同促进下夺取 $3 b$-位亚 甲基质子, 再经电子转移脱氢开环, 在 $3 b$-位上形成苯 亚胺结构 $\mathbf{C}$, 其 $\alpha, \beta$-不饱和亚胺与水分子首先发生迈克 尔加成, 先给出 $\beta$-差而亚胺 $\mathbf{D}$ (由此得到紫红卟啉 $\mathbf{5}$ ), 其 $3 \mathrm{c}$-位上的苯亚胺再经水解转化成醇酮成分 $\mathbf{E}$ (由此得到 紫红卟啉 6), 最后酸催化脱水构成芳甲酰乙基取代基团 $\mathbf{F}$ (由此得到紫红卟啉 7). 紫红卟啉碳架的形成原因主要
是由于芳胺和三氟化硼的酸碱协同作用，脱去 D-子环 的两个 $\beta$-位氢原子而氧化成芳香性的吡咯环, 图 2 框内 标识了上述反应过程中的脱氢历程.

\section{2 红紫素-18 及其衍生物的 12-位非甲基化反应}

利用二氢卟吩色基上的 3-位乙烯基、3-位甲酰基和 外接环羰基等取代基团的各种化学反应是修饰叶绿素 大环分子的重要方法 ${ }^{[8]}$, 但是关于 12 -位甲基的结构转 换却报道甚少，本文基于本研究室前期的研究工 作 $^{[9 \sim 11]}$, 选择红紫素-18 为研究对象, 进一步扩展叶绿 素降解产物的 12-位官能化的研究. 红紫素-18 二酰亚胺 的 C(12)-甲基与环上其它惰性甲基相比(如 2-, 7-和 18位甲基), 存在着潜在的化学反应活性, 由于红紫素-18 及其二酰亚胺衍生物的基本碳架同样属于二氢卟吩环 系, 图 3 中 $1 \mathbf{a}$ 是其众多芳香性氮杂轮烯共轭体系中的最 为稳定的存在方式, 即二氮杂-18-轮烯结构, 其芳香性 $\pi$-体系的环电流分配均匀, 中心氮上氢原子又相距最 远，因而可以使得四吡咯大环分子的内能保持在最低状 态. 作为二氢卟吩大环的另一种存在方式三氮杂-17-轮 烯(1b), 虽然 $\pi$-体系也满足 $4 n+2$ 规则而具有芳香性, 但其氮杂轮烯缺少对称性, 因而其芳香性程度相对较 低, 在大环分子芳香性氮杂轮烯中贡献也要低于 1a. 三 氮杂-17-轮烯 $(\mathbf{1 b})$ 离域区域并没有涵盖 $\mathbf{C}$ 环的 $\beta$-位, 在 碱性条件下, 吡咯子环中的碳碳双键与外接环中两个羰 基形成烯酮结构，其 12-位甲基可以通过插烯作用而表 现出与羰基 $\alpha$-位相似的反应活性. 在碱性条件下, 红紫 素-18 甲酯或者其二酰亚胺的外接环酐羰基通过 C(12)$\mathrm{C}(13)$ 位或者 C(14)-C(15)位双键的插烯作用，活化了 12位甲基的反应活性，使 12-位甲基上的氢原子呈现出与 羰基 $\alpha$-氢相类似的化学性质，即 12 -位甲基质子在
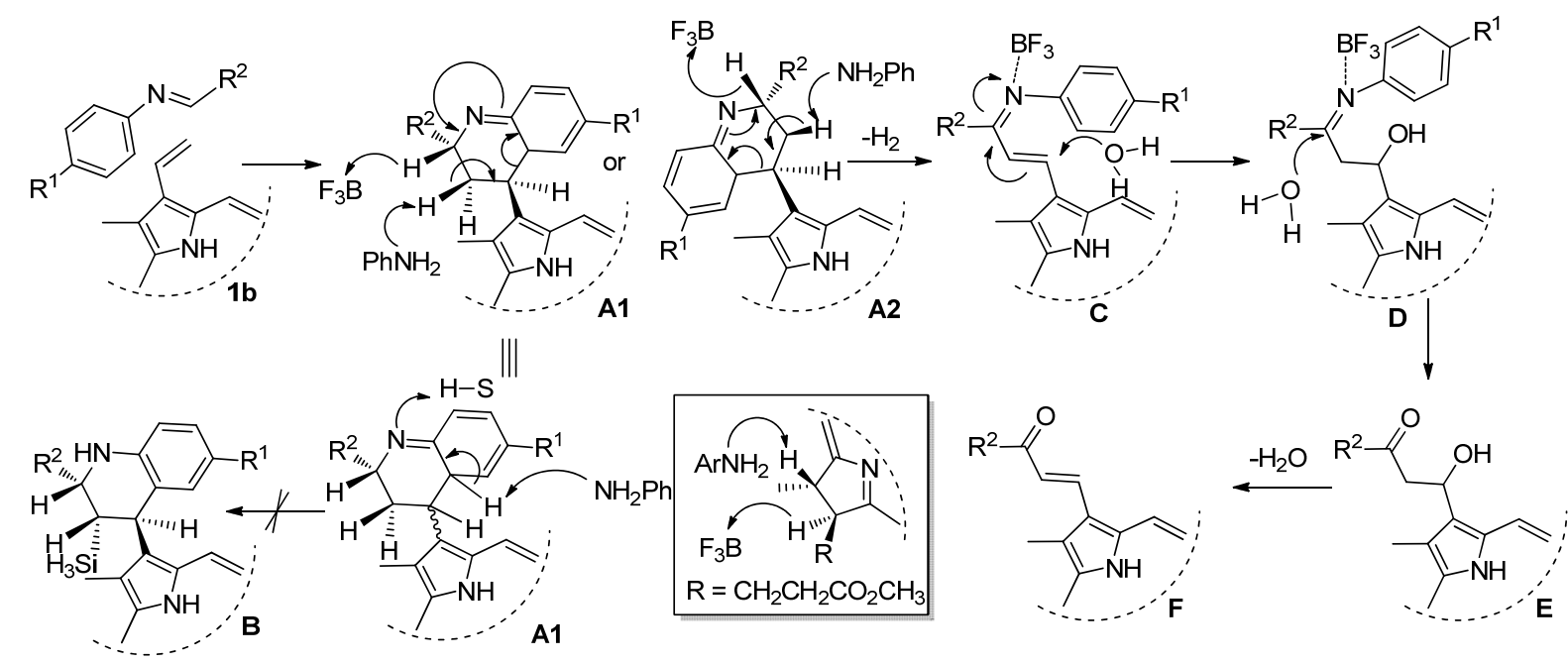

图 $2 \mathrm{~N}$-甲氧基红紫素-18 二酰亚胺 $\mathbf{1 b}$ 与 $N$-苯基亚胺的 Povarov 反应

Figure 2 The Povarov reaction of $N$-methyoxyl purpurin imide with $N$-phenylimide 

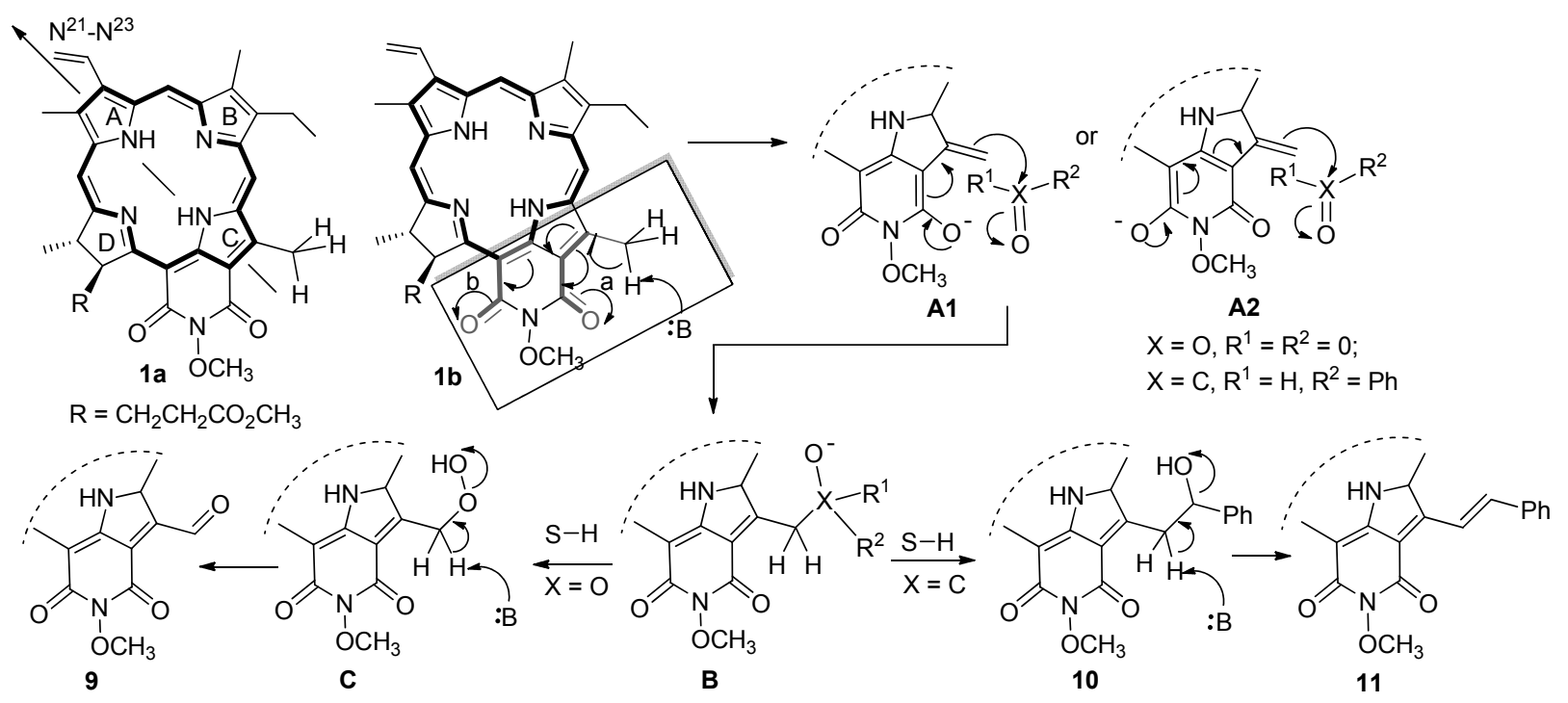

图 3 红紫素-18 二酰亚胺芳香性氮杂轮烯结构及其 12-位甲基的化学反应活性

Figure 3 The aromatic aza-annulene structure of purpurin-18 imide and chemical activity on C(12)-methyl group

碱存在时能够发生电离, 所得负电荷通过 $\mathbf{a}$ 和 $\mathbf{b}$ 两条共 轭途径转移. 所形成的两个烯醇式氧负离子 $\mathbf{A 1}$ 和 $\mathbf{A 2}$, 在空气氧化或者羟醛缩合反应中, 首先对含氧双键实施 亲核加成并转化成氧负离子中间体 $\mathbf{B}$, 当 $\mathrm{X}$ 为氧原子的 时候, 夺取反应体系中的质子构成含有过氧键的二氢卟 吩中间体 $\mathbf{C}$, 在碱的继续作用下脱去一分子水并转化成 二氢卟吩醛 $(\boldsymbol{9})$. 在羟醛缩合反应中 $(X=C)$, 中间体 $\mathbf{B}$ 捕 获质子后形成亲核加成的四面体中间体 10 , 再经酸催 化脱水得 12-苯甲叉基取代的红紫素-18 二亚酰胺(11).

\section{2 结论}

具有叶绿素基本碳架的红紫素-18 及其二酰亚胺的 六元外接环的环上羰基对芳香性氮杂轮烯的环电流分 布产生了明显的影响, 因而在一定程度上改变了大环分 子的化学反应活性. 通过 3-位乙烯基的 1,3-偶极和杂 Diles-Alder 环加成反应, 在 3-位上建立芳杂环、芳酰基 和芳烷基等取代结构; 利用 20-meso-位和 12-位甲基的 多电子性, 可以在二氢卟吩色基上顺利完成亲电取代、 空气氧化和类羟醛缩合等经典的化学反应, 并在周环的 12-和 20-位上引进了不同的取代基团, 扩展了叶绿素降 解产物在 $\mathrm{N}^{21}-\mathrm{N}^{23}$ 轴向实施官能团转化的范围, 为获取 新型叶绿素类二氢卟吩衍生物提供了便利的合成途径.

\section{3 实验部分}

\section{1 仪器与试剂}

元素分析用 Perkin-Elmer 2400 型元素分析仪测定; IR 用 Perkin-Elmer 1730 型红外分光光度仪测定 $(\mathrm{KBr}$ 压 片); UV-Vis 用 UV-160A 型紫外分光光度计测定; ${ }^{1} \mathrm{H}$
NMR 用 Brucker ARX-300 型核磁共振仪测定, 内标为 TMS. 所用试剂均为分析纯或化学纯红紫素-18 甲酯 (1a)和红紫素-18 二亚酰胺甲酯(1b)按文献[12]制备.

\section{$3.23-\left[5^{\prime}(R / S)-\left(3^{\prime}\right.\right.$-对氯苯基-4',5-二氢异噁唑基)]-3-去 乙烯基红紫素-18 甲酯(2a)和 3-甲酰基-3-去乙烯基红紫 素-18 甲酯(3)的合成}

向含有 $120 \mathrm{mg}(0.680 \mathrm{mmol}) \mathrm{NBS}$ 的 $2 \mathrm{~mL}$ 干燥二氯 甲烷中一次性加入 $106 \mathrm{mg}(0.700 \mathrm{mmol})$ 对氯苯甲醛肜, 室温摚拌反应 $20 \mathrm{~min}$ 溴化结束, 将溶有 $436 \mathrm{mg}(0.754$ $\mathrm{mmol}) \mathbf{1 a}$ 的 $3 \mathrm{~mL}$ 干燥的二氯甲烷倒入上述混合液中, 在 $15 \mathrm{~min}$ 内滴加溶解于 $2 \mathrm{~mL}$ 干燥的二氯甲烷的 $72 \mathrm{mg}$ $(0.717 \mathrm{mmol})$ 三乙胺, 氮气保护, 室温搅拌 $24 \mathrm{~h}$, 加入 20 $\mathrm{mL}$ 二氯甲烷和 $25 \mathrm{~mL}$ 水分层, 分出有机相后水洗两次, 无水硫酸钠干燥, 减压浓缩, 剩余物经硅胶柱层析分离 [洗脱剂: $V$ (丙酮 $): V($ 苯 $)=1: 10$ ], 分别得到 $254 \mathrm{mg}$ 红 色固体 2a (0.347 mmol, 46\%) 和 $13 \mathrm{mg}$ 红色固体 $3(0.023$ mmol, 3\%). 2a: m.p. $218 \sim 221{ }^{\circ} \mathrm{C}$; UV-vis $\left(\mathrm{CHCl}_{3}\right) \lambda_{\max }$ $\left[\varepsilon /\left(\mathrm{L}^{\circ} \mathrm{mol}^{-1} \cdot \mathrm{cm}^{-1}\right)\right]: 410\left(1.15 \times 10^{5}\right), 476\left(4.79 \times 10^{3}\right), 505$ $\left(5.70 \times 10^{3}\right), 541\left(1.87 \times 10^{4}\right), 698\left(1.06 \times 10^{5}\right) \mathrm{nm} ;{ }^{1} \mathrm{H}$ NMR $\left(400 \mathrm{MHz}, \mathrm{CDCl}_{3}\right) \delta$ : -2.49 (br s, $1 \mathrm{H}, \mathrm{NH}$ ), -0.05 (br s, $1 \mathrm{H}, \mathrm{NH}), 1.68$ (1.67) (t, $J=7.6 \mathrm{~Hz}, 3 \mathrm{H}$, $\left.8 \mathrm{~b}-\mathrm{CH}_{3}\right), 1.87$ (d, $J=7.2 \mathrm{~Hz}, 3 \mathrm{H}, 18-\mathrm{CH}_{3}$ ), $2.25 \sim 2.43$ (m, $2 \mathrm{H}, 17 \mathrm{a}+17 \mathrm{~b}-\mathrm{H}), 2.68 \sim 2.84(\mathrm{~m}, 2 \mathrm{H}, 17 \mathrm{a}+17 \mathrm{~b}-\mathrm{H}), 3.69$ (3.68) (q, $J=7.6 \mathrm{~Hz}, 2 \mathrm{H}, 8 \mathrm{a}-\mathrm{H}), 3.14,3.53$ (3.54), 3.59, 3.78 (3.76) (each s, each $\left.3 \mathrm{H}, \mathrm{OCH}_{3}+\mathrm{CH}_{3}\right), 4.10$ (4.09) (d, $J=16.9 \mathrm{~Hz}, 1 \mathrm{H}, 3 \mathrm{~b}-\mathrm{H}), 4.36$ (4.38) (dd, $J=16.9,10.2 \mathrm{~Hz}$, $1 \mathrm{H}, 3 \mathrm{~b}-\mathrm{H}$ ), 4.70 (q, $J=7.2 \mathrm{~Hz}, 1 \mathrm{H}, 18-\mathrm{H}), 5.14$ (d, $J=8.9$ $\mathrm{Hz}, 1 \mathrm{H}, 17-\mathrm{H}), 7.23$ (7.24) (t, $J=10.0 \mathrm{~Hz}, 1 \mathrm{H}, 3 \mathrm{a}-\mathrm{H}), 7.52$ 
(d, $J=8.4 \mathrm{~Hz}, 2 \mathrm{H}, \mathrm{PhH}), 7.89$ (7.88) $(\mathrm{d}, J=8.4 \mathrm{~Hz}, 2 \mathrm{H}$, $\mathrm{PhH}$ ), 9.01, 9.75 (9.72), 9.83 (9.82) (each s, each 1H, meso-H); IR (KBr) v: $3389(\mathrm{~N}-\mathrm{H}), 2914(\mathrm{C}-\mathrm{H}), 1734(\mathrm{C}=$ O), $1617(\mathrm{C}=\mathrm{C}), 1547$ (chlorin skeleton), 1480, 1345, 1215, 1024, $982 \mathrm{~cm}^{-1}$; EI-MS m/z: $733.3\left(\mathrm{M}+\mathrm{H}^{+}\right)$. Anal. calcd for $\mathrm{C}_{41} \mathrm{H}_{38} \mathrm{ClN}_{5} \mathrm{O}_{6}$ : C 67.25, H 5.23, N 9.56; found $\mathrm{C}$ 67.47, H 5.06, N 9.29. 3: 物理常数与分析数据均与文献 [12]一致.

3.3 3-[5'(R/S)-(3'-对甲氧苯基-4',5.-二氢异噁唑基)]-3去乙烯基红紫素-18 甲酯(2b)和 3-甲酰基-3-去乙烯基红 紫素-18 甲酯(3)的合成

以对甲氧基苯甲醛肜替代对氯苯甲醛肜，按照合成 $2 \mathbf{a}$ 的方法合成得到 50\%的 $2 \mathbf{b}$ 和 4\%的 3. $2 \mathbf{b}$ : m.p. 221

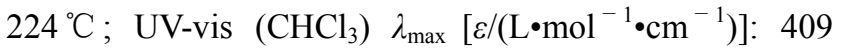
$\left(1.14 \times 10^{5}\right), \quad 478 \quad\left(4.12 \times 10^{3}\right), \quad 504 \quad\left(5.35 \times 10^{3}\right), \quad 541$ $\left(1.81 \times 10^{4}\right), 698\left(1.07 \times 10^{5}\right) \mathrm{nm} ;{ }^{1} \mathrm{H}$ NMR $(400 \mathrm{MHz}$. $\left.\mathrm{CDCl}_{3}\right) \delta:-0.15$ (br s, $\left.1 \mathrm{H}, \mathrm{NH}\right),-0.10$ (br s, $1 \mathrm{H}, \mathrm{NH}$ ), 1.63 (t, $\left.J=7.6 \mathrm{~Hz}, 3 \mathrm{H}, 8 \mathrm{~b}-\mathrm{CH}_{3}\right), 1.74$ (d, $J=7.2 \mathrm{~Hz}, 3 \mathrm{H}$, $\left.18-\mathrm{CH}_{3}\right), 1.94 \sim 2.01(\mathrm{~m}, 1 \mathrm{H}, 17 \mathrm{a}+17 \mathrm{~b}-\mathrm{H}), 2.47 \sim 2.61$ (m, 2H, 17a+17b-H), 2.72 2.77 (m, 1H, 17a+17b-H), $3.61(\mathrm{q}, J=7.6 \mathrm{~Hz}, 2 \mathrm{H}, 8 \mathrm{a}-\mathrm{H}), 3.00,3.40,3.59,3.79$, 3.93(each s, each $\left.3 \mathrm{H}, \mathrm{OCH}_{3}+\mathrm{CH}_{3}\right), 4.28 \sim 4.32(\mathrm{~m}, 2 \mathrm{H}$, 3b-H), 4.40 (q, $J=7.2 \mathrm{~Hz}, 1 \mathrm{H}, 18-\mathrm{H}), 5.20$ (d, $J=8.4 \mathrm{~Hz}$, $1 \mathrm{H}, 17-\mathrm{H}), 7.00$ (t, $J=10.0 \mathrm{~Hz}, 1 \mathrm{H}, 3 \mathrm{a}-\mathrm{H}), 7.04$ (d, $J=8.7$ $\mathrm{Hz}, 2 \mathrm{H}, \mathrm{PhH}), 7.86$ (d, J=8.7 Hz, 2H, $\mathrm{PhH}), 8.59,9.46$, 9.62 (each s, each 1H, meso-H); IR (KBr) v: $3434(\mathrm{~N}-\mathrm{H})$, 2925, $2858(\mathrm{C}-\mathrm{H}), 1739,1701(\mathrm{C}=\mathrm{O}), 1623(\mathrm{C}=\mathrm{C})$, 1562 (chlorin skeleton), 1463, 1373, 1228, 1168, 1029, 906, $732 \mathrm{~cm}^{-1}$; EI-MS m/z: $728.4\left(\mathrm{M}+\mathrm{H}^{+}\right)$. Anal. calcd for $\mathrm{C}_{42} \mathrm{H}_{41} \mathrm{~N}_{5} \mathrm{O}_{7}$ : C 69.31, H 5.68, N 9.62; found $\mathrm{C} 69.40$, $\mathrm{H}$ 5.74, N 9.50.

3.43 3-[5'(R/S)-(3'-2"-吡啶基-4',5.-二氢异噁唑基)]-3去乙烯基红紫素-18 甲酯(2c)和 3-甲酰基-3-去乙烯基红 紫素-18 甲酯(3)的合成

以 2-吡啶醛肜替代对氯苯甲醛肜, 按照合成 $2 \mathbf{a}$ 的 方法合成得到 $44 \%$ 的 $2 \mathrm{c}$ 和 $2 \%$ 的 3. 2c: m.p. 224

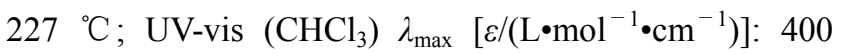
$\left(1.11 \times 10^{5}\right), \quad 474 \quad\left(4.05 \times 10^{3}\right), \quad 504 \quad\left(5.17 \times 10^{3}\right), \quad 542$ $\left(1.71 \times 10^{4}\right), 638\left(4.98 \times 10^{3}\right), 698\left(1.09 \times 10^{5}\right) \mathrm{nm} ;{ }^{1} \mathrm{H}$ NMR (400 MHz. $\left.\mathrm{CDCl}_{3}\right) \delta:-0.16$ (br s, 1H, NH), 0.11 (br s, 1H, NH), 1.61 (t, $J=7.6 \mathrm{~Hz}, 3 \mathrm{H}, 8 \mathrm{~b}-\mathrm{CH}_{3}$ ), 1.75 $\left(\mathrm{d}, J=7.2 \mathrm{~Hz}, 3 \mathrm{H}, 18-\mathrm{CH}_{3}\right), 1.94 \sim 2.03(\mathrm{~m}, 1 \mathrm{H}, 17 \mathrm{a}+$ $17 \mathrm{~b}-\mathrm{H}), 2.42 \sim 2.48(\mathrm{~m}, 2 \mathrm{H}, 17 \mathrm{a}+17 \mathrm{~b}-\mathrm{H}), 2.73 \sim 2.78(\mathrm{~m}$, $1 \mathrm{H}, 17 \mathrm{a}+17 \mathrm{~b}-\mathrm{H}$ ), 3.55 (q, $J=7.6 \mathrm{~Hz}, 2 \mathrm{H}, 8 \mathrm{a}-\mathrm{H}$ ), 2.98, $3.37,3.60,3.73$ (each s, each $3 \mathrm{H}, \mathrm{OCH}_{3}+\mathrm{CH}_{3}$ ), 4.28 $4.32(\mathrm{~m}, 2 \mathrm{H}, 3 \mathrm{~b}-\mathrm{H}), 4.41$ (q, $J=7.2 \mathrm{~Hz}, 1 \mathrm{H}, 18-\mathrm{H}), 5.20$ $(\mathrm{d}, J=8.3 \mathrm{~Hz}, 1 \mathrm{H}, 17-\mathrm{H}), 7.02 \sim 708(\mathrm{~m}, 1 \mathrm{H}, 3 \mathrm{a}-\mathrm{H}), 7.90$ (d, $J=8.7 \mathrm{~Hz}, 2 \mathrm{H}, \mathrm{PhH}), 8.37$ (d, $J=8.7 \mathrm{~Hz}, 2 \mathrm{H}, \mathrm{PhH}$ ), 8.61, 9.41, 9.54 (each s, each $1 \mathrm{H}$, meso-H); IR (KBr) $v$ : $3409(\mathrm{~N}-\mathrm{H}), 2932,2912(\mathrm{C}-\mathrm{H}), 1734(\mathrm{C}=\mathrm{O}), 1642$ $(\mathrm{C}=\mathrm{C}), 1522$ (chlorin skeleton), 1432, 1383, 1249, 1165, 1039, $962 \mathrm{~cm}^{-1}$; EI-MS m/z: $699.4\left(\mathrm{M}+\mathrm{H}^{+}\right)$. Anal. calcd for $\mathrm{C}_{40} \mathrm{H}_{38} \mathrm{~N}_{6} \mathrm{O}_{6}$ : C 68.75, H 5.48, N 12.03; found C 68.80, H 5.61, N 12.21.

3.53 -[2'(S),4'(R)-1'-甲基-2"-氧代-5"-氯-吲哚啉-3"--螺 -2'-吡咯烷基]-红紫素-18 甲酯(4a/4b)和 3-[2'(R),4'(R)1'-甲基-2"-氧代 5"-氯-吲哚啉-3"-螺-2'-吡咯烷基]-红紫 素-18 甲酯( $4 b / 4 a)$ 的合成

将 $60 \mathrm{mg}$ 红紫素-18 甲酯 $1 \mathrm{a}(0.104 \mathrm{mmol})$ 溶解于 5 $\mathrm{mL}$ 干燥的甲苯中, 先后加入 $11 \mathrm{mg}$ 甲基甘氨酸 $(0.124$ $\mathrm{mmol}$ )和 $19 \mathrm{mg}$ 对氯靛红 $(0.104 \mathrm{mmol})$, 在 $110{ }^{\circ} \mathrm{C}$ 搅拌回 流 $7 \mathrm{~h}$ 后, 向反应体系中加入 $10 \mathrm{~mL}$ 冷水并搅拌 $10 \mathrm{~min}$, 再加入 $20 \mathrm{~mL}$ 二氯甲烷分层, 水层用二氯甲烷萃取(15 $\mathrm{mL} \times 2)$, 合并有机层, 用无水硫酸钠干燥, 减压浓缩, 剩余物经硅胶柱层析分离 [洗脱剂: $V$ (石油醚) : $V$ (乙酸 乙酯 $)=4: 1$ ], 分别得到 $17 \mathrm{mg}$ 红棕色固体 $\mathbf{4 a} / \mathbf{4 b}(0.022$ $\mathrm{mmol}, 21 \%$ )和 $20 \mathrm{mg}$ 红棕色固体 $4 \mathbf{b} / \mathbf{4 a}$ (0.026 mmol, $25 \%)$.

4a/4b: mp. $191 \sim 194{ }^{\circ} \mathrm{C}$; UV-vis $\left(\mathrm{CH}_{2} \mathrm{Cl}_{2}\right) \lambda_{\max }$

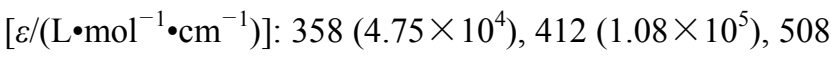
$\left(4.54 \times 10^{2}\right), 544\left(2.59 \times 10^{4}\right), 640\left(1.07 \times 10^{4}\right), 694$ $\left(6.37 \times 10^{4}\right) \mathrm{nm} ;{ }^{1} \mathrm{H}$ NMR $\left(400 \mathrm{MHz} . \mathrm{CDCl}_{3}\right) \delta:-0.18$ (br s, 1H, NH), 0.07 (br s, 1H, NH), 1.62 (t, $J=7.6 \mathrm{~Hz}, 3 \mathrm{H}$, $\left.8 \mathrm{a}-\mathrm{CH}_{3}\right), 1.69$ (d, $\left.J=7.0 \mathrm{~Hz}, 3 \mathrm{H}, 18-\mathrm{CH}_{3}\right), 1.84 \sim 2.08$ (m, $1 \mathrm{H}, 17 \mathrm{a}+17 \mathrm{~b}-\mathrm{H}), 2.45\left(\mathrm{~s}, 3 \mathrm{H}, \mathrm{NCH}_{3}\right), 2.28 \sim 2.50(\mathrm{~m}, 2 \mathrm{H}$, $17 \mathrm{a}+17 \mathrm{~b}-\mathrm{H}), 2.66 \sim 2.74(\mathrm{~m}, 1 \mathrm{H}, 17 \mathrm{a}+17 \mathrm{~b}-\mathrm{H}), 2.95 \sim$ $3.05\left(\mathrm{~m}, 1 \mathrm{H}, 3^{\prime}-\mathrm{H}\right), 3.48 \sim 3.62\left(\mathrm{~m}, 1 \mathrm{H}, 3{ }^{\prime}-\mathrm{H}\right), 3.09,3.33$, 3.59 (3.58), 3.60 (each s, each $\left.3 \mathrm{H}, \mathrm{CH}_{3}+\mathrm{OCH}_{3}\right), 3.62$ (q, $J=7.6 \mathrm{~Hz}, 2 \mathrm{H}, 8 \mathrm{~b}-\mathrm{H}), 3.65 \sim 3.80\left(\mathrm{~m}, 1 \mathrm{H}, 5^{\prime}-\mathrm{H}\right), 4.33$ (4.29) (q, $J=7.3 \mathrm{~Hz}, 1 \mathrm{H}, 18-\mathrm{H}), 5.13$ (5.12) (d, $J=7.3 \mathrm{~Hz}$, $1 \mathrm{H}, 17-\mathrm{H}), 4.07 \sim 4.18$ (m, 1H, 5'-H), 5.25 (br s, 1H, 4'-H), 6.23 (6.19) (d, $J=8.3 \mathrm{~Hz}, 1 \mathrm{H}, \mathrm{PhH}), 6.78$ (br s, 1H, PhH), 7.09 (7.05) (d, $J=8.3 \mathrm{~Hz}, 1 \mathrm{H}, \mathrm{PhH}), 7.89,8.47,9.42$ (each $\mathrm{s}$, each $1 \mathrm{H}$, meso-H), 9.15 (br s, $1 \mathrm{H}, \mathrm{NH})$; IR (KBr) v: 3431, $3135(\mathrm{~N}-\mathrm{H}), 2924,2854(\mathrm{C}-\mathrm{H}), 1695,1736(\mathrm{C}=$ O), 1654, $1637(\mathrm{C}=\mathrm{C}), 1560$ (chlorin skeleton), 1508, 1400, 1267, $1070 \mathrm{~cm}^{-1}$; EI-MS m/z: $788.3\left(\mathrm{MH}^{+}\right)$. Anal. calcd for $\mathrm{C}_{44} \mathrm{H}_{44} \mathrm{ClN}_{6} \mathrm{O}_{6}$ : C 67.12, $\mathrm{H}$ 5.51, N 10.67; found C 67.28, H 5.32, N 10.57.

4b/4a: m.p. $192 \sim 195{ }^{\circ} \mathrm{C}$; UV-vis $\left(\mathrm{CH}_{2} \mathrm{Cl}_{2}\right) \lambda_{\max }$ 


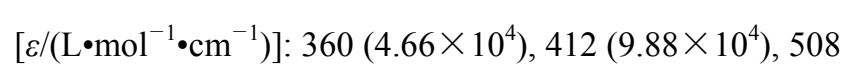
$\left(4.45 \times 10^{2}\right), 544\left(2.54 \times 10^{4}\right), 638\left(9.63 \times 10^{4}\right), 694$ $\left(6.21 \times 10^{4}\right) \mathrm{nm} ;{ }^{1} \mathrm{H}$ NMR $\left(400 \mathrm{MHz} . \mathrm{CDCl}_{3}\right) \delta:-0.11$ (br s, $1 \mathrm{H}, \mathrm{NH}), 0.15$ (br s, $1 \mathrm{H}, \mathrm{NH}), 1.60(\mathrm{t}, J=7.6 \mathrm{~Hz}, 3 \mathrm{H}$, $\left.8 \mathrm{a}-\mathrm{CH}_{3}\right), 1.67$ (d, $\left.J=7.0 \mathrm{~Hz}, 3 \mathrm{H}, 18-\mathrm{CH}_{3}\right), 1.90 \sim 2.06(\mathrm{~m}$, $1 \mathrm{H}, 17 \mathrm{a}+17 \mathrm{~b}-\mathrm{H}), 2.52\left(\mathrm{~s}, 3 \mathrm{H}, \mathrm{NCH}_{3}\right), 2.32 \sim 2.52(\mathrm{~m}, 2 \mathrm{H}$, $17 \mathrm{a}+17 \mathrm{~b}-\mathrm{H}), 2.54 \sim 2.70(\mathrm{~m}, 1 \mathrm{H}, 17 \mathrm{a}+17 \mathrm{~b}-\mathrm{H}), 2.95 \sim$ $3.05\left(\mathrm{~m}, 1 \mathrm{H}, 3^{\prime}-\mathrm{H}\right), 3.14 \sim 3.40$ (m, 1H, 3'-H), 3.43 (q, $J=$ $7.6 \mathrm{~Hz}, 2 \mathrm{H}, 8 \mathrm{~b}-\mathrm{H}$ ), 3.09, 3.19, 3.59, 3.61 (each s, each 3H, $\left.\mathrm{CH}_{3}+\mathrm{OCH}_{3}\right), 3.92 \sim 4.04\left(\mathrm{~m}, 2 \mathrm{H}, 5{ }^{\prime}-\mathrm{H}\right), 4.34$ (4.33) (q, $J=7.3 \mathrm{~Hz}, 1 \mathrm{H}, 18-\mathrm{H}), 5.19(5.14)(\mathrm{d}, J=7.7 \mathrm{~Hz}, 1 \mathrm{H}$, 17-H), 5.25 (br s, 1H, 4'-H), 6.30 (br s, 1H, PhH), 6.56 (6.54) (d, $J=8.2 \mathrm{~Hz}, 1 \mathrm{H}, \mathrm{PhH}), 6.80$ (6.75) (d, $J=8.2 \mathrm{~Hz}$, $1 \mathrm{H}, \mathrm{PhH}$ ), 7.80, 8.43, 9.69 (each s, each $1 \mathrm{H}$, meso-H), 9.29 (br s, 1H, NH); IR (KBr) v: 3446, $3131(\mathrm{~N}-\mathrm{H}), 2900(\mathrm{C}-$ H), 1736, $1701(\mathrm{C}=\mathrm{O}), 1655,1611(\mathrm{C}=\mathrm{C}) 1560,1541$ (chlorin skeleton), 1400, 1309, 1257, $1079 \mathrm{~cm}^{-1}$; EI-MS $m / z$ : 787.7. Anal. calcd for $\mathrm{C}_{44} \mathrm{H}_{44} \mathrm{ClN}_{6} \mathrm{O}_{6}$ : $\mathrm{C} 67.12$, H 5.51, $\mathrm{N} 10.67$; found C 67.37, H 5.70, N 10.60 .

3.6 $N$-甲氧基-3-[1' $(R / S)$-1'-羟基-3'-对甲氧苯亚胺基3'-对硝苯丙基]-3-去乙烯基紫红卟啉二酰亚胺(5)、N-甲 氧基-3-[1'(R/S)-1'-羟基-2'-对硝苯甲酰基乙基]-3-去乙 烯基紫红卟啉二酰亚胺甲酯(6)和 $N$-甲氧基-3b-对硝基 苯甲酰基红紫素-18 二酰亚胺甲酯(7)的合成

将 $89 \mathrm{mg} \mathrm{N}$-甲氧基红紫素-18 酰亚胺甲酯(1b) (0.294 mmol)溶解于 $4 \mathrm{~mL}$ 甲苯中, 加入 $24 \mathrm{mg}$ 对硝基苯 甲醛 $(0.160 \mathrm{mmol})$ 和 $20 \mathrm{mg}$ 对甲氧基苯胺 $(0.160 \mathrm{mmol})$ 以后, 再加入 $0.1 \mathrm{~mL}$ 三氟化嗍乙醚溶液, $110{ }^{\circ} \mathrm{C}$ 摚拌回 流 $10 \mathrm{~h}$, 反应结束后向反应混合物中加入 $15 \mathrm{~mL}$ 冷水, 分出有机层, 水层用二氯甲烷萃取 $(15 \mathrm{~mL} \times 2)$. 合并有 机层, 水洗后用无水硫酸钠干燥, 减压浓缩, 剩余物经 硅胶柱层析分离 [洗脱剂: $V$ (石油醚) $: V($ 乙酸乙酯 $)=$ $4: 1$ ], 分别得到 $14 \mathrm{mg}$ 绿色固体 $5(0.015 \mathrm{mmol}, 5 \%)$ 、 $16 \mathrm{mg}$ 绿色固体 6 (0.020 mmol, 7\%) 和 $55 \mathrm{mg}$ 黑红色固体 7 (0.073 mmol, 25\%).

5: $256 \sim 259{ }^{\circ} \mathrm{C}$; UV-vis $\left(\mathrm{CH}_{2} \mathrm{Cl}_{2}\right) \lambda_{\max }\left[\varepsilon /\left(\mathrm{L}^{\circ} \mathrm{mol}^{-1}\right.\right.$. $\left.\left.\mathrm{cm}^{-1}\right)\right]: 434\left(9.78 \times 10^{4}\right), 492\left(1.00 \times 10^{3}\right), 559$ $\left(4.89 \times 10^{3}\right), 617\left(7.82 \times 10^{3}\right), 667\left(1.21 \times 10^{4}\right) \mathrm{nm} ;{ }^{1} \mathrm{H}$ NMR (400 MHz, $\left.\mathrm{CDCl}_{3}\right) \delta$ : -2.60 (br s, $1 \mathrm{H}, \mathrm{NH}$ ), -2.40 (br s, $1 \mathrm{H}, \mathrm{NH}), 1.78(1.76)(\mathrm{t}, J=7.6 \mathrm{~Hz}, 3 \mathrm{H}$, $\left.8 \mathrm{a}-\mathrm{CH}_{3}\right), 2.81 \sim 2.90(\mathrm{~m}, 2 \mathrm{H}, 17 \mathrm{a}+17 \mathrm{~b}-\mathrm{H}), 3.32 \sim 3.40$ (m, 2H, 17a +17b-H), 3.22 (3.21), 3.26, 3.56, 3.57, 3.59, 3.77, 4.41 (each s, each $3 \mathrm{H}, \mathrm{CH}_{3}+\mathrm{OCH}_{3}$ ), 3.98 (t, $J=7.6$ $\mathrm{Hz}, 2 \mathrm{H}, 8 \mathrm{~b}-\mathrm{H}), 4.25 \sim 4.36(\mathrm{~m}, 2 \mathrm{H}, 3 \mathrm{~b}-\mathrm{H}), 5.59 \sim 5.70(\mathrm{~m}$, $2 \mathrm{H}, 3 \mathrm{a}-\mathrm{H}+\mathrm{PhH}), 6.77 \sim 6.82(\mathrm{~m}, 1 \mathrm{H}, \mathrm{PhH}), 6.99$ (d, $J=$
$8.1 \mathrm{~Hz}, 1 \mathrm{H}, \mathrm{PhH}), 7.48 \sim 7.57$ (m, 3H, PhH), $7.58 \sim 7.68$ (m, 2H, PhH), 9.79 (9.75) 9.86 (9.83), 9.95 (9.92) (each s, each 1H, meso-H); IR (KBr) v: $3438(\mathrm{~N}-\mathrm{H}), 2936,2912$ $(\mathrm{C}-\mathrm{H}), 1733,1697(\mathrm{C}=\mathrm{O}), 1608(\mathrm{C}=\mathrm{C}), 1549$ (chlorin skeleton), 1487, 1432, 1343, 1203, 1153, 1081, $981 \mathrm{~cm}^{-1}$; EI-MS $m / z$ : $878.5\left(\mathrm{M}+\mathrm{H}^{+}\right)$. Anal. calcd for $\mathrm{C}_{49} \mathrm{H}_{47} \mathrm{~N}_{7} \mathrm{O}_{9}$ : C 67.04, H 5.40, N 11.17; found C 67.11, H 5.26, N 11.29.

6: $241 \sim 243{ }^{\circ} \mathrm{C}$; UV-vis $\left(\mathrm{CH}_{2} \mathrm{Cl}_{2}\right) \lambda_{\max }\left[\varepsilon /\left({\mathrm{L} \cdot \mathrm{mol}^{-1}}^{-}\right.\right.$ $\left.\mathrm{cm}^{-1}\right)$ ]: $434\left(1.11 \times 10^{4}\right), 490\left(8.97 \times 10^{3}\right), 557(5.01 \times$ $\left.10^{3}\right), 615\left(8.81 \times 10^{3}\right), 664\left(9.96 \times 10^{3}\right) \mathrm{nm} ;{ }^{1} \mathrm{H}$ NMR $(400$ $\left.\mathrm{MHz}, \mathrm{CDCl}_{3}\right) \delta:-2.36$ (br s, $\left.1 \mathrm{H}, \mathrm{NH}\right),-2.16(\mathrm{br} \mathrm{s}, 1 \mathrm{H}$, $\mathrm{NH}), 1.76\left(\mathrm{t}, J=7.6 \mathrm{~Hz}, 3 \mathrm{H}, 8 \mathrm{a}-\mathrm{CH}_{3}\right), 2.33(\mathrm{~d}, J=7.5 \mathrm{~Hz}$, $2 \mathrm{H}, 17 \mathrm{~b}-\mathrm{H}), 3.30$ (d, $J=7.5 \mathrm{~Hz}, 2 \mathrm{H}, 17 \mathrm{a}-\mathrm{H}), 3.33,3.43$, 3.57, 3.60, 3.88, 4.34 (each s, each $3 \mathrm{H}, \mathrm{CH}_{3}+\mathrm{OCH}_{3}$ ), 3.95 (t, $J=7.6 \mathrm{~Hz}, 2 \mathrm{H}, 8 \mathrm{~b}-\mathrm{H}), 4.27 \sim 4.36(\mathrm{~m}, 2 \mathrm{H}, 3 \mathrm{~b}-\mathrm{H})$, $5.50 \sim 5.70(\mathrm{~m}, 1 \mathrm{H}, 3 \mathrm{a}-\mathrm{H}), 7.20(\mathrm{~d}, J=7.8 \mathrm{~Hz}, 2 \mathrm{H}, \mathrm{PhH})$, $7.61(\mathrm{~d}, J=7.8 \mathrm{~Hz}, 2 \mathrm{H}, \mathrm{PhH}), 9.90,9.70,9.97$ (each s, each 1H, meso-H); IR (KBr) v: $3398(\mathrm{~N}-\mathrm{H}), 2960,2927$ $(\mathrm{C}-\mathrm{H}), 1737,1709(\mathrm{C}=\mathrm{O}), 1618(\mathrm{C}=\mathrm{C}), 1552$ (chlorin skeleton), 1456, 1222, 1164, 993, $729 \mathrm{~cm}^{-1}$; EI-MS $\mathrm{m} / z$ : $773.3\left(\mathrm{M}+\mathrm{H}^{+}\right)$. Anal. calcd for $\mathrm{C}_{42} \mathrm{H}_{40} \mathrm{~N}_{6} \mathrm{O}_{9}: \mathrm{C} 65.28, \mathrm{H}$ 5.22, N 10.87; found C 65.49, H 5.11, N 11.01 .

7: $205 \sim 208{ }^{\circ} \mathrm{C}$; UV-vis $\left(\mathrm{CH}_{2} \mathrm{Cl}_{2}\right) \lambda_{\max }\left[\varepsilon /\left(\mathrm{L}^{\circ} \mathrm{mol}^{-1} \bullet\right.\right.$ $\left.\mathrm{cm}^{-1}\right)$ ]: $420\left(1.05 \times 10^{4}\right), 427\left(9.23 \times 10^{3}\right), 556(4.78 \times$ $\left.10^{3}\right), 667\left(9.96 \times 10^{3}\right), 714\left(1.13 \times 10^{3}\right) \mathrm{nm} ;{ }^{1} \mathrm{H}$ NMR $(400$ $\left.\mathrm{MHz}, \mathrm{CDCl}_{3}\right) \delta: 0.14$ (br s, $\left.1 \mathrm{H}, \mathrm{NH}\right), 0.16$ (br s, $1 \mathrm{H}, \mathrm{NH}$ ), $1.68\left(\mathrm{t}, J=7.6 \mathrm{~Hz}, 3 \mathrm{H}, 8 \mathrm{a}-\mathrm{CH}_{3}\right), 1.74(\mathrm{~d}, J=7.2 \mathrm{~Hz}$, $\left.18-\mathrm{CH}_{3}\right), 1.90 \sim 2.06(\mathrm{~m}, 1 \mathrm{H}, 17 \mathrm{a}+17 \mathrm{~b}-\mathrm{H}), 2.42 \sim 2.56$ (m, 2H, 17a+17b-H), 2.77 2.87 (m, 1H, 17a +17b-H), 3.66 (q, $J=7.5 \mathrm{~Hz}, 2 \mathrm{H}, 8 \mathrm{a}-\mathrm{H}$ ), 3.19, 3.47, 3.60, 3.83, 4.36 (each s, each $3 \mathrm{H}, \mathrm{CH}_{3}+\mathrm{OCH}_{3}$ ), 4.39 (q, $J=7.3 \mathrm{~Hz}, 1 \mathrm{H}$, $18-\mathrm{H}), 5.29$ (d, $J=8.4 \mathrm{~Hz}, 1 \mathrm{H}, 17-\mathrm{H}), 7.54(\mathrm{~d}, J=16.1 \mathrm{~Hz}$, 1H, 3b-H), 7.58 (d, $J=7.8 \mathrm{~Hz}, 2 \mathrm{H}, \mathrm{PhH}), 7.71$ (d, $J=7.8$ $\mathrm{Hz}, 2 \mathrm{H}, \mathrm{PhH}), 8.47$ (d, $J=16.1 \mathrm{~Hz}, 1 \mathrm{H}, 3 \mathrm{a}-\mathrm{H}), 8.80,9.35$, 9.63 (each s, each 1H, meso-H); IR (KBr) v: $3456(\mathrm{~N}-\mathrm{H})$, 2960, $2867(\mathrm{C}-\mathrm{H}), 1743,1703(\mathrm{C}=\mathrm{O}), 1645,1608(\mathrm{C}=$ C), 1535 (chlorin skeleton), 1461, 1402, 1309, 1143, 1074 $\mathrm{cm}^{-1}$; EI-MS $m / z$ : $757.4\left(\mathrm{M}+\mathrm{H}^{+}\right)$. Anal. calcd for $\mathrm{C}_{42} \mathrm{H}_{40} \mathrm{~N}_{6} \mathrm{O}_{8}$ : C 66.66, H 5.33, N 11.10; found C 66.80, H 5.18, N 11.20 .

\subsection{N-(吡啶-4-甲基)-红紫素-18 二酰亚胺甲酯(8)的合 成}

将 $120 \mathrm{mg} \mathrm{1b}(0.198 \mathrm{mmol})$ 溶解于 $15 \mathrm{~mL}$ 吡啶中, 再加入 $1 \mathrm{~mL}$ 4-氨甲基吡啶, 室温搅拌反应 $5 \mathrm{~h}$ 后, 加入 $200 \mathrm{~mL}$ 盐酸水溶液 $(20 \mathrm{~mL}$ 浓盐酸 $+180 \mathrm{~mL}$ 水), 然后用 
二氯甲烷萃取 $(80 \mathrm{~mL} \times 2)$, 合并有机相并水洗除酸, 用 无水硫酸钠干燥, 减压浓缩, 剩余物经硅胶柱层析分离 [洗脱剂: $V$ (石油醚) $: V$ (乙酸乙酯 $)=4: 1$ ], 得到 $91 \mathrm{mg}$ 红色固体 8 (0.136 mmol), 产率 69\%. m.p. 201 203 ${ }^{\circ} \mathrm{C}$; UV-vis $\left(\mathrm{CH}_{2} \mathrm{Cl}_{2}\right) \lambda_{\max }\left[\varepsilon /\left(\mathrm{L} \cdot \mathrm{mol}^{-1} \cdot \mathrm{cm}^{-1}\right)\right]: 420(9.78 \times$ $\left.10^{4}\right), 511\left(9.92 \times 10^{3}\right), 551\left(5.04 \times 10^{3}\right), 650\left(7.66 \times 10^{3}\right)$, $707\left(3.26 \times 10^{4}\right) \mathrm{nm} ;{ }^{1} \mathrm{H} \mathrm{NMR}\left(400 \mathrm{MHz}, \mathrm{CDCl}_{3}\right) \delta:-$ 0.08 (br s, 1H, NH), 0.05 (br s, 1H, NH), 1.61 (t, $J=7.6$ $\left.\mathrm{Hz}, 3 \mathrm{H}, 8 \mathrm{a}-\mathrm{CH}_{3}\right), 1.71\left(\mathrm{~d}, J=7.3 \mathrm{~Hz}, 3 \mathrm{H}, 18-\mathrm{CH}_{3}\right), 1.90 \sim$ $2.00(\mathrm{~m}, 1 \mathrm{H}, 17 \mathrm{a}+17 \mathrm{~b}-\mathrm{H}), 2.33 \sim 2.43(\mathrm{~m}, 2 \mathrm{H}, 17 \mathrm{a}+$ 17b-H), $2.64 \sim 2.76(\mathrm{~m}, 1 \mathrm{H}, 17 \mathrm{a}+17 \mathrm{~b}-\mathrm{H}), 3.08,3.32,3.54$, 3.75 (each s, each $3 \mathrm{H}, \mathrm{CH}_{3}+\mathrm{OCH}_{3}$ ), 3.54 (t, $J=7.6 \mathrm{~Hz}$, $2 \mathrm{H}, 8 \mathrm{~b}-\mathrm{H}), 4.37$ (q, $J=7.2 \mathrm{~Hz}, 1 \mathrm{H}, 18-\mathrm{H}), 5.33$ (d, $J=9.2$ $\mathrm{Hz}, 17-\mathrm{H}), 5.68$ (s, 2H, $\mathrm{NCH}_{2}$ ), 6.13 (dd, $J=11.5,1.2 \mathrm{~Hz}$, $1 \mathrm{H}$, cis-3b-H), 6.25 (dd, $J=17.8,1.2 \mathrm{~Hz}$, trans-3b-H), 7.58 (d, $J=7.5 \mathrm{~Hz}, 2 \mathrm{H}$, Pyr-H), 7.83 (dd, $J=17.8,11.5 \mathrm{~Hz}$, 3a-H), 8.60 (d, J=7.5 Hz, 2H, Pyr-H), 8.54, 9.25, 9.49 (each s, each 1H, meso-H); IR (KBr) v: $3477(\mathrm{~N}-\mathrm{H})$, 2927, $2856(\mathrm{C}-\mathrm{H}), 1741,1712(\mathrm{C}=\mathrm{O}), 1633(\mathrm{C}=\mathrm{C})$, 1556 (chlorin skeleton), 1467, 1380, 1261, 1101, 806 $\mathrm{cm}^{-1}$; EI-MS m/z: $669.3\left(\mathrm{M}+\mathrm{H}^{+}\right)$. Anal. calcd for $\mathrm{C}_{40} \mathrm{H}_{40^{-}}$ $\mathrm{N}_{6} \mathrm{O}_{4}$ : C 71.84, H 6.03, N 12.57; found C 71.80, H 6.21, N 12.79 .

\section{$3.8 N$-甲氧基-12-甲酰基红紫素-18 二酰亚胺甲酯(9) 的合成}

将 $79 \mathrm{mg}$ 红紫素-18 衍生物 $\mathbf{1 b}(0.130 \mathrm{mmol})$ 溶解于 $20 \mathrm{~mL}$ 甲醇中, 搅拌下加入氢氧化锂水溶液 $(0.4 \mathrm{~g} \mathrm{LiOH}$ $+5 \mathrm{~mL}$ 水), 室温条件下开口剧烈搅拌 $4 \mathrm{~h}$ 后, 用乙酸将 反应液的酸性调至 $\mathrm{pH}$ 值为 3 , 加入 $50 \mathrm{~mL}$ 二氯甲烷和 5 $\mathrm{mL}$ 水分层, 分出有机相, 水相用二氯甲烷萃取 (50 $\mathrm{mL} \times 3)$, 合并有机相, 水洗至中性, 用无水硫酸钠干燥, 浓缩至 $10 \mathrm{~mL}$, 加入过量的自制重氮甲烷, 振摇后(约 1 $\min$ ) 快速用冰乙酸处理未反应的重氮甲烷, 减压蒸除溶 剂, 剩余物经硅胶柱层析分离 [洗脱剂: $V$ (石油醚) : $V($ 乙酸乙酯 $)=9: 2]$, 得到 $25 \mathrm{mg}$ 红色固体 $9(0.040$ mmol, 31\%). m.p. 253 256 ${ }^{\circ} \mathrm{C}$; UV-vis $\left(\mathrm{CH}_{2} \mathrm{Cl}_{2}\right) \lambda_{\max }$

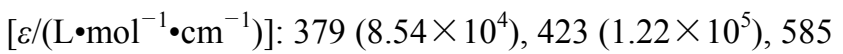
$\left(3.29 \times 10^{4}\right), 659\left(2.93 \times 10^{4}\right), 715\left(5.37 \times 10^{4}\right) \mathrm{nm} ;{ }^{1} \mathrm{H}$ NMR $\left(\mathrm{CDCl}_{3}\right) \delta$ : -0.06 (br s, $\left.1 \mathrm{H}, \mathrm{NH}\right), 0.80$ (br s, $1 \mathrm{H}$, $\mathrm{NH}), 1.60\left(\mathrm{t}, J=7.6 \mathrm{~Hz}, 3 \mathrm{H}, 8-\mathrm{CH}_{3}\right), 1.67$ (d, $J=7.3 \mathrm{~Hz}$, $\left.3 \mathrm{H}, 18-\mathrm{CH}_{3}\right), 1.85 \sim 1.95(\mathrm{~m}, 1 \mathrm{H}, 17 \mathrm{a}+17 \mathrm{~b}-\mathrm{H}), 2.36 \sim$ $2.45(\mathrm{~m}, 1 \mathrm{H}, 17 \mathrm{a}+17 \mathrm{~b}-\mathrm{H}), 2.48 \sim 2.58(\mathrm{~m}, 1 \mathrm{H}, 17 \mathrm{a}+$ $17 \mathrm{~b}-\mathrm{H}), 2.76 \sim 2.86(\mathrm{~m}, 1 \mathrm{H}, 17 \mathrm{a}+17 \mathrm{~b}-\mathrm{H}), 2.97,3.22,3.62$, 4.35 (each s, each $\left.3 \mathrm{H}, \mathrm{CH}_{3}+\mathrm{OCH}_{3}\right), 3.49$ (q, $J=7.6 \mathrm{~Hz}$, 2H, 8a-H), 4.18 (q, $J=7.4 \mathrm{~Hz}, 1 \mathrm{H}, 18-\mathrm{H}), 5.07$ (d, $J=9.1$
Hz, 1H, 17-H), 6.16 (d, J=11.5 Hz, 1H, cis-3b-H), 6.23 (d, $J=17.8 \mathrm{~Hz}, 1 \mathrm{H}$, trans-3b-H), 7.68 (dd, $J=17.8,11.5$ $\mathrm{Hz}, 1 \mathrm{H}, 3 \mathrm{a}-\mathrm{H}$ ), 8.16, 8.79, 10.21 (each s, each $1 \mathrm{H}$, meso-H), 11.78 (s, 1H, CHO); IR (KBr) v: $3458(\mathrm{~N}-\mathrm{H})$, $2926(\mathrm{C}-\mathrm{H}), 1739,1700(\mathrm{C}=\mathrm{O}), 1616(\mathrm{C}=\mathrm{C}), 1551$ (chlorin skeleton), 1461, 1226, 1039, $993 \mathrm{~cm}^{-1}$; EI-MS $m / z: 622.3\left(\mathrm{M}+\mathrm{H}^{+}\right)$. Anal. calcd for $\mathrm{C}_{35} \mathrm{H}_{35} \mathrm{~N}_{5} \mathrm{O}_{6}: \mathrm{C} 67.62$, H 5.67, N 11.27; found C 67.79, H 5.50, N 11.14.

\section{$3.9 \mathrm{~N}$-甲氧基-12-(2'-羟基-2'-苯基乙基)-红紫素-18 二 酰亚胺(10)的合成}

将 $46 \mathrm{mg}$ 金属钠溶解于 $10 \mathrm{~mL}$ 甲醇中, 先后加入溶 有 $128 \mathrm{mg} 1 \mathrm{~b}(0.211 \mathrm{mmol})$ 的 $5 \mathrm{~mL}$ 甲醇和 $0.4 \mathrm{~mL}$ 新蒸 馏的苯甲醛, 氮气保护, $35{ }^{\circ} \mathrm{C}$ 下搅拌反应, $6 \mathrm{~h}$ 反应结 束, 先后向反应体系加入 $20 \mathrm{~mL}$ 水和 $25 \mathrm{~mL}$ 二氯甲烷, 分出有机相, 用二氯甲烷萃取水相 $(15 \mathrm{~mL} \times 3)$, 合并有 机层并用无水硫酸钠干燥, 减压浓缩, 将所得粘稠状混 合物用硅胶柱层析 [洗脱剂: $V($ 苯 $): V($ 丙酮 $)=10 ： 1$ ] 分 离, 得到 $65 \mathrm{mg}$ 红色色固体 $\mathbf{1 0}$ (0.091 mmol). 产率 43\%, m.p. $217 \sim 220{ }^{\circ} \mathrm{C}$; UV-vis $\left(\mathrm{CH}_{2} \mathrm{Cl}_{2}\right) \lambda_{\max }\left[\varepsilon /\left(\mathrm{L} \cdot \mathrm{mol}^{-1}\right.\right.$ • $\left.\mathrm{cm}^{-1}\right)$ ]: $410\left(1.04 \times 10^{5}\right), 513\left(6.36 \times 10^{3}\right), 554(8.78 \times$ $\left.10^{3}\right), \quad 640\left(7.15 \times 10^{3}\right), 706\left(4.94 \times 10^{4}\right) \mathrm{nm} ;{ }^{1} \mathrm{H}$ NMR $\left(\mathrm{CDCl}_{3}\right) \delta: 0.46$ (br s, $\left.1 \mathrm{H}, \mathrm{NH}\right), 0.61$ (br s, $\left.1 \mathrm{H}, \mathrm{NH}\right), 1.60$ (1.59) (t, $\left.J=7.6 \mathrm{~Hz}, 3 \mathrm{H}, 8-\mathrm{CH}_{3}\right), 1.72(\mathrm{~d}, J=7.2 \mathrm{~Hz}, 3 \mathrm{H}$, $\left.18-\mathrm{CH}_{3}\right), 1.90 \sim 2.05(\mathrm{~m}, 1 \mathrm{H}, 17 \mathrm{a}+17 \mathrm{~b}-\mathrm{H}), 2.35 \sim 2.56$ (m, 1H, 17a+17b-H), $2.72 \sim 2.86(\mathrm{~m}, 1 \mathrm{H}, 17 \mathrm{a}+17 \mathrm{~b}-\mathrm{H})$, $2.74 \sim 2.83$ (m, 1H, 17a+17b-H), 3.09, 3.30, 3.60 (3.59), 4.39 (each s, each $3 \mathrm{H}, \mathrm{CH}_{3}+\mathrm{OCH}_{3}$ ), $3.46(\mathrm{t}, J=7.6 \mathrm{~Hz}$, $2 \mathrm{H}, 8 \mathrm{a}-\mathrm{H}), 4.32$ (q, $J=7.2 \mathrm{~Hz}, 1 \mathrm{H}, 18-\mathrm{H}), 5.23$ (d, $J=8.6$ $\mathrm{Hz}, 1 \mathrm{H}, 17-\mathrm{H}), 6.15$ (d, J=11.6 Hz, 1H, cis-3b-H), 6.26 $(\mathrm{d}, J=17.8 \mathrm{~Hz}, 1 \mathrm{H}$, trans $-3 \mathrm{~b}-\mathrm{H}), 7.18 \sim 7.26(\mathrm{~m}, 1 \mathrm{H}$, $\mathrm{PhH}$ ), 7.32 (7.29) (t, $J=7.3 \mathrm{~Hz}, 2 \mathrm{H}, \mathrm{PhH}), 7.60$ (7.66) (d, $J=7.4 \mathrm{~Hz}, 2 \mathrm{H}, \mathrm{PhH}), 7.82(\mathrm{dd}, J=17.8,11.6 \mathrm{~Hz}, 1 \mathrm{H}$, 3a-H), 8.45, 9.19 (9.14), 9.20 (each s, each 1H, meso-H); IR (KBr) v: $3454(\mathrm{~N}-\mathrm{H}), 2962(\mathrm{C}-\mathrm{H}), 1728,1693(\mathrm{C}=$ O), $1610(\mathrm{C}=\mathrm{C}), 1552$ (chlorin skeleton), 1450, 1222, 1153, 1039, $840 \mathrm{~cm}^{-1}$; EI-MS m/z: $714.4\left(\mathrm{M}+\mathrm{H}^{+}\right)$. Anal. calcd for $\mathrm{C}_{42} \mathrm{H}_{43} \mathrm{~N}_{5} \mathrm{O}_{6}$ : C 70.67, $\mathrm{H} \mathrm{6.07,} \mathrm{N} \mathrm{9.81;} \mathrm{found} \mathrm{C}$ 70.51, H 6.27, N 9.97.

\section{$3.10 \mathrm{~N}$-甲氧基-12-苯甲叉基红紫素-18 二亚酰胺甲酯 (11)的合成}

将 $40 \mathrm{mg} 10(0.056 \mathrm{mmol})$ 溶解于 $10 \mathrm{~mL}$ 干燥的二氯 甲烷中, 再加入研碎的 $20 \mathrm{mg}$ 对甲苯磺酸, 于 $35{ }^{\circ} \mathrm{C}$ 下 搅拌反应 $24 \mathrm{~h}$, 加入 $15 \mathrm{~mL}$ 冷水再搅拌 $5 \mathrm{~min}$, 分出有机 层, 再水洗一次, 用无水硫酸钠干燥, 减压除去溶剂, 剩余物用硅胶柱层析[洗脱剂: $V($ 苯 $): V($ 丙酮 $)=10 ： 1]$ 
分离反应混合物, 得 $25 \mathrm{mg}$ 红色固体 $11(0.036 \mathrm{mmol})$, 产率 65\%. m.p. 212 215 ${ }^{\circ} \mathrm{C}$; UV-vis $\left(\mathrm{CH}_{2} \mathrm{Cl}_{2}\right) \lambda_{\max }$

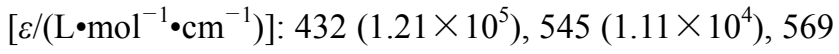
$\left(1.38 \times 10^{4}\right), 656\left(1.69 \times 10^{4}\right), 730\left(6.23 \times 10^{4}\right) \mathrm{nm} ;{ }^{1} \mathrm{H}$ NMR $\left(\mathrm{CDCl}_{3}\right) \delta: 0.47$ (br s, $\left.1 \mathrm{H}, \mathrm{NH}\right), 0.53($ br s, $1 \mathrm{H}, \mathrm{NH})$, 1.55 (t, $\left.J=7.6 \mathrm{~Hz}, 3 \mathrm{H}, 8-\mathrm{CH}_{3}\right), 1.73(\mathrm{~d}, J=7.3 \mathrm{~Hz}, 3 \mathrm{H}$, $\left.18-\mathrm{CH}_{3}\right), 1.90 \sim 2.01(\mathrm{~m}, 1 \mathrm{H}, 17 \mathrm{a}+17 \mathrm{~b}-\mathrm{H}), 2.43 \sim 2.58$ (m, $2 \mathrm{H}, 17 \mathrm{a}+17 \mathrm{~b}-\mathrm{H}), 2.80 \sim 2.87(\mathrm{~m}, 1 \mathrm{H}, 17 \mathrm{a}+17 \mathrm{~b}-\mathrm{H})$, 3.02, 3.26, 3.60, 3.62, 4.36 (each s, each $3 \mathrm{H}, \mathrm{CH}_{3}+$ $\mathrm{OCH}_{3}$ ), 3.38 (q, $\left.J=7.6 \mathrm{~Hz}, 2 \mathrm{H}, 8 \mathrm{a}-\mathrm{H}\right), 4.31$ (q, $J=7.4 \mathrm{~Hz}$, $1 \mathrm{H}, 18-\mathrm{H}), 5.25$ (dd, $J=9.6,2.2 \mathrm{~Hz}, 1 \mathrm{H}, 17-\mathrm{H}), 6.12$ (d, $J=11.5 \mathrm{~Hz}$, cis-3b-H), 6.22 (d, $J=17.5 \mathrm{~Hz}$, trans $-3 \mathrm{~b}-\mathrm{H})$, 7.44 (t, $J=7.3 \mathrm{~Hz}, 1 \mathrm{H}, \mathrm{PhH}), 7.53(\mathrm{t}, J=7.3 \mathrm{~Hz}, 2 \mathrm{H}$, $\mathrm{PhH}), 7.72$ (d, $J=16.5 \mathrm{~Hz}, 12 \mathrm{~b}-\mathrm{H}), 7.78$ (dd, $J=17.8,11.5$ $\mathrm{Hz}, 1 \mathrm{H}, 3 \mathrm{a}-\mathrm{H}), 7.81$ (t, $J=7.3 \mathrm{~Hz}, 2 \mathrm{H}, \mathrm{PhH}), 8.68$ (d, $J=$ $16.5 \mathrm{~Hz}, 1 \mathrm{H}, 12 \mathrm{a}-\mathrm{H}), 8.42,9.32,9.42$ (each s, each 1H, meso-H); IR (KBr) v: $3436(\mathrm{~N}-\mathrm{H}), 2924(\mathrm{C}-\mathrm{H}), 1726$, $1678(\mathrm{C}=\mathrm{O}), 1622(\mathrm{C}=\mathrm{C}), 1585$ (chlorin skeleton), 1373, 1283, 1014, $912 \mathrm{~cm}^{-1}$; EI-MS m/z: $696.3\left(\mathrm{M}-\mathrm{H}^{+}\right)$. Anal. calcd for $\mathrm{C}_{42} \mathrm{H}_{41} \mathrm{~N}_{5} \mathrm{O}_{5}$ : C 72.50, H 5.94, N 10.07; found $\mathrm{C}$ 72.77, H 6.05, N 10.19 .

\section{$3.11 \mathrm{~N}$-甲氧基-20-硝基红紫素-18 二亚酰胺甲酯(12) 的合成}

将 $117 \mathrm{mg} \mathbf{1 b}(0.193 \mathrm{mmol})$ 溶解于 $10 \mathrm{~mL}$ 三氯甲烷 中, 滴加溶解于 $2 \mathrm{~mL}$ 乙酸的 $0.5 \mathrm{~mL}$ 发烟硝酸, 室温摚 拌 $5 \mathrm{~h}$, 反应结束后倒入 $150 \mathrm{~g}$ 碎冰中, 分层后用二氯甲 烷萃取 $(50 \mathrm{~mL} \times 3)$, 合并有机层, 水洗两次, 用无水硫 酸钠干燥, 减压除去溶剂, 用硅胶柱层析 [洗脱剂: $V$ (苯) $: V($ 丙酮 $)=10 ： 1]$ 分离反应混合物, 得 $48 \mathrm{mg}$ 红 色固体 12(0.073 mmol), 产率 38\%. m.p. 202 205 ${ }^{\circ} \mathrm{C}$; UV-vis $\left(\mathrm{CH}_{2} \mathrm{Cl}_{2}\right) \lambda_{\max }\left[\varepsilon /\left(\mathrm{L} \cdot \mathrm{mol}^{-1} \cdot \mathrm{cm}^{-1}\right)\right]$ : $420(1.17 \times$ $\left.10^{5}\right), 535\left(1.21 \times 10^{4}\right), 561\left(1.40 \times 10^{4}\right), 646\left(1.77 \times 10^{4}\right)$, $715\left(5.23 \times 10^{4}\right) \mathrm{nm} ;{ }^{1} \mathrm{H}$ NMR $\left(\mathrm{CDCl}_{3}\right) \delta:-1.78(\mathrm{br} \mathrm{s}$, $1 \mathrm{H}, \mathrm{NH}), 0.04$ (br s, $1 \mathrm{H}, \mathrm{NH}), 1.75(\mathrm{~d}, J=7.3 \mathrm{~Hz}, 3 \mathrm{H}$, $\left.18-\mathrm{CH}_{3}\right), 1.76\left(\mathrm{t}, J=7.6 \mathrm{~Hz}, 3 \mathrm{H}, 8-\mathrm{CH}_{3}\right), 2.19 \sim 2.24(\mathrm{~m}$, $1 \mathrm{H}, \quad 17 \mathrm{a}+17 \mathrm{~b}-\mathrm{H}), \quad 2.33 \sim 2.41(\mathrm{~m}, 1 \mathrm{H}, 17 \mathrm{a}+17 \mathrm{~b}-\mathrm{H})$, $2.56 \sim 2.64(\mathrm{~m}, 1 \mathrm{H}, 17 \mathrm{a}+17 \mathrm{~b}-\mathrm{H}), 2.68 \sim 2.76(\mathrm{~m}, 1 \mathrm{H}$, $17 \mathrm{a}+17 \mathrm{~b}-\mathrm{H}$ ), 3.40, 3.60, 3.64, 3.65 (each s, each $3 \mathrm{H}, \mathrm{CH}_{3}$ $\left.+\mathrm{OCH}_{3}\right), 3.81(\mathrm{q}, J=7.6 \mathrm{~Hz}, 2 \mathrm{H}, 8 \mathrm{a}-\mathrm{H}), 4.91$ (q, $J=7.4$ $\mathrm{Hz}, 1 \mathrm{H}, 18-\mathrm{H}$ ), 5.20 (dd, $J=9.0,2.5 \mathrm{~Hz}, 1 \mathrm{H}, 17-\mathrm{H}), 6.25$ (d, $J=17.9 \mathrm{~Hz}$, trans-3b-H), 6.39 (d, $J=10.3 \mathrm{~Hz}$, cis-3b-H), 7.99 (dd, $J=17.9,10.3 \mathrm{~Hz}, 1 \mathrm{H}, 3 \mathrm{a}-\mathrm{H}), 9.62$, 9.97 (each s, each 1H, meso-H); IR (KBr) v: $3517(\mathrm{~N}-\mathrm{H})$, 2966, $2883(\mathrm{C}-\mathrm{H}), 1733,1701(\mathrm{C}=\mathrm{O}), 1616(\mathrm{C}=\mathrm{C})$, 1554 (chlorin skeleton), 1392, 1307, 1189, 1101, 1027, 925 $\mathrm{cm}^{-1}$; EI-MS $m / z$ : $653.3\left(\mathrm{M}+\mathrm{H}^{+}\right)$. Anal. calcd for $\mathrm{C}_{35} \mathrm{H}_{36} \mathrm{~N}_{6} \mathrm{O}_{7}$ : C 64.41, H 5.56, N 12.88; found C 64.36, H 5.40, N 12.79 .

\section{$3.12 \mathrm{~N}$-甲氧基-12-甲酰基-20-氯红紫素-18 二亚酰胺 甲酯(13)的合成}

将 $80 \mathrm{mg} 9$ (0.129 mmol)溶解于 $15 \mathrm{~mL}$ 二氯甲烷中, 搅拌下慢慢滴加含有 $50 \mathrm{mg} N$-氯代丁二酰亚胺 $(0.374$ $\mathrm{mmol})$ 的 $5 \mathrm{~mL}$ 甲醇溶液, $1.5 \mathrm{~h}$ 滴加完毕, TLC 监测决定 反应时间, 约 $4 \mathrm{~h}$ 反应结束. 先后向反应体系加入 $25 \mathrm{~mL}$ 水和 $25 \mathrm{~mL}$ 二氯甲烷, 分出有机层, 水层用二氯甲烷萃 取 $(10 \mathrm{~mL} \times 2)$, 合并有机层, 用无水硫酸钠干燥, 减压 除去溶剂, 用硅胶柱层析[洗脱剂: $V($ 苯 $): V($ 丙酮 $)=8$ : 1]分离反应混合物, 得 $41 \mathrm{mg}$ 红色固体 13 (0.063 mmol), 产率 49\%. m.p. 226 229 ${ }^{\circ} \mathrm{C}$; UV-vis $\left(\mathrm{CH}_{2} \mathrm{Cl}_{2}\right) \lambda_{\text {max }}$

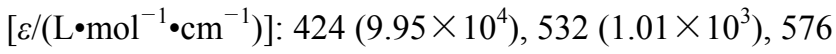
$\left(1.49 \times 10^{3}\right), 661\left(2.13 \times 10^{4}\right), 722\left(4.76 \times 10^{4}\right) \mathrm{nm} ;{ }^{1} \mathrm{H}$ NMR $\left(\mathrm{CDCl}_{3}\right) \delta: 0.05($ br s, $1 \mathrm{H}, \mathrm{NH}), 0.82(\mathrm{br} \mathrm{s}, 1 \mathrm{H}, \mathrm{NH})$, $1.52\left(\mathrm{~d}, J=7.0 \mathrm{~Hz}, 3 \mathrm{H}, 18-\mathrm{CH}_{3}\right), 1.60$ (t, $J=7.6 \mathrm{~Hz}, 3 \mathrm{H}$, $\left.8-\mathrm{CH}_{3}\right), 1.89 \sim 1.02(\mathrm{~m}, 1 \mathrm{H}, 17 \mathrm{a}+17 \mathrm{~b}-\mathrm{H}), 2.25 \sim 2.37(\mathrm{~m}$, $1 \mathrm{H}, 17 \mathrm{a}+17 \mathrm{~b}-\mathrm{H}), 2.52 \sim 2.63(\mathrm{~m}, 1 \mathrm{H}, 17 \mathrm{a}+17 \mathrm{~b}-\mathrm{H})$, $2.77 \sim 2.87(\mathrm{~m}, 1 \mathrm{H}, 17 \mathrm{a}+17 \mathrm{~b}-\mathrm{H}), 2.99,3.39,3.60,4.38$ (each s, each $3 \mathrm{H}, \mathrm{CH}_{3}+\mathrm{OCH}_{3}$ ), $3.51(\mathrm{q}, J=7.6 \mathrm{~Hz}, 2 \mathrm{H}$, $8 \mathrm{a}-\mathrm{H}), 4.68$ (q, $J=7.0 \mathrm{~Hz}, 1 \mathrm{H}, 18-\mathrm{H}), 5.12$ (dd, $J=9.2,2.8$ $\mathrm{Hz}, 1 \mathrm{H}, 17-\mathrm{H}), 6.10(\mathrm{dd}, J=17.8,1.4 \mathrm{~Hz}$, trans $-3 \mathrm{~b}-\mathrm{H})$, 6.25 (dd, $J=11.5,1.4 \mathrm{~Hz}$, cis-3b-H), 7.65 (dd, $J=17.8$, $11.5 \mathrm{~Hz}, 1 \mathrm{H}, 3 \mathrm{a}-\mathrm{H}$ ), 8.90, 10.22 (each s, each $1 \mathrm{H}$, meso-H), 11.78 (s, 1H, CHO); IR (KBr) v: 3409, $3303(\mathrm{~N}-\mathrm{H})$, 2960, $2866(\mathrm{C}-\mathrm{H}), 1726,1703(\mathrm{C}=\mathrm{O}), 1647(\mathrm{C}=\mathrm{C})$, 1550 (chlorin skeleton), 1460, 1380, 1271, 1205, 1143, $1070 \mathrm{~cm}^{-1}$; EI-MS $m / z: 657.4\left(\mathrm{M}+\mathrm{H}^{+}\right)$. Anal. calcd for $\mathrm{C}_{35} \mathrm{H}_{34} \mathrm{ClN}_{5} \mathrm{O}_{6}$ : C 64.07, H 5.22, N 10.67; found C 64.16, H 5.30, N 10.74 .

辅助材料(Supporting Information) 新化合物的核磁 共振氢谱和质谱图. 这些材料可以免费从本刊网站 (http://sico-journal.cn/)上下载.

\section{References}

[1] (a) Chen, Y. H.; Li, G. L.; Pandey, R. K. Curr. Org. Chem. 2004, 8, 1105.

(b) Wang, J.-J. Chin. J. Org. Chem. 2005, 25, 1353 (in Chinese).

(王进军, 有机化学, 2005, 25, 1353.)

(c) Yang, Z.; Wang, Z.; Liu, Y.; Xu, X.-S.; Qi, C.-X.; Wang, J.-J. Chin. J. Org. Chem. 2013, 33, 116 (in Chinese).

(杨泽, 王振, 刘洋, 徐希森, 祁彩霞, 王进军, 有机化学, 2013, 33, 116.)

[2] (a) Kozyrev, A.; Ethirajan, M.; Chen, P.; Ohkubo, K.; Robin- 
son, G. C.; Berkigia, K. M.; Fukuzumi, S.; Kadish, K. M.; Pandey, J. J. Org. Chem. 2012, 77, 10260.

(b) Pavlov, V. Y.; Ponomarev, G. V. Chem. Heterocycl. Compd. 2004, 40, 393.

(c) Kozyrey, A. N.; Chen, Y.-H.; Goswami, L. N.; Tabaczynaki, W. A.; Pandey, R. K. J. Org. Chem. 2006, 71, 1949.

(d) Liu, R.-R.; Wang, L.-M.; Yin, J.-G.; Wu, J.; Liu, C.; Zhang, P.; Wang, J.-J. Chin. J. Org. Chem. 2012, 32, 318 (in Chinese).

(刘舟手, 王鲁敏, 金英学, 武进, 刘超, 王朋, 王进军, 有 机化学, 2012, 32, 318.)

[3] (a) Wang, J.-J.; Yin, Y.-F.; Yang, Z. J. Iran. Chem. Soc. 2013, 10, 583 .

(b) Wang, L.-M.; Wang, P.; Liu, C.; Jin, Y.-X.; Wang, J.-J. Chin. J. Org. Chem. 2012, 32, 1700 (in Chinese).

(王鲁敏, 王朋, 刘超, 金英学, 王进军, 有机化学, 2012, 32, 1700.)

(c) Yin, J.-G.; Li, Y.-W.; Li, J.-Z.; Zhang, Q.; Pei, W.; Wang, J.-J. Chin. J. Org. Chem. 2011, 31, 1213 (in Chinese). (殷军港, 李韵伟, 李家柱, 张千, 裴文, 王进军, 有机化 学, 2011, 31, 1213.)

(d) Li, J.-Z.; Wang, J.-J.; Yoon, L.; Cui, B.-C.; Shim, Y.-K. Bioorg. Med. Chem. Lett. 2012, 22, 1846.

(e) Li, J.-Z.; Zhang, P.; Yao, N.-N.; Zhao, L.-L.; Wang, J.-J.; Shim, Y.-K. Tetrahedron Lett. 2014, 55, 1086

[4] (a) Wang, P.; Yang, Z.; Li, J.-Z.; Yao, N.-N.; Wang, J.-J. Chin. J. Org. Chem. 2012, 32, 368 (in Chinese).

(王朋, 杨泽, 李家柱, 姚楠楠, 王进军, 有机化学, 2012, 32, 368.)

(b) Grysshuk, A.; Chen, Y.; Goswami, L. N.; Pandey, S.; Missert, J. R.; Qhulchanskyy, T.; Potter, W.; Prasad, P. P.; Oseroff, A.; Pandey, R. K. J. Med. Chem. 2006, 49, 1874.

(c) Pandey, S. K.; Sajjad, M.; Chen, Y.; Pandey, A.; Missert, J. R.; Batt, C.; Yao, R.; Nabi, H. A.; Oseroff, A. R.; Pandey, R. K. Bioconijuate Chem. 2009, 20, 274.

[5] Smith, C. D.; Gavrilyuk, J. I.; Lough, A. J.; Batey, R. A. J.
Org. Chem. 2010, 75, 72.

[6] (a) Houk, K. N.; Sins, J.; Duck, R. E. J. Am. Chem. Soc. 1973, 95, 7287.

(b) Houk, K. N.; Sins, J.; Watts, C. R. J. Am. Chem. Soc. 1973, 95, 7301 .

[7] (a) Glusshkov, V. A.; Tolstikov, A. G. Russ. Chem. Rev. 2008, 77, 137.

(b) Povarov, L. S. Russ. Chem. Rev. 1967, 36, 656.

[8] (a) Yu, S.-S.; Xu, X.-S.; Liu, Y.; Li, J.-Z.; Jin, Y.-X.; Qi, C.-X.; Wang, J.-J. Chin. J. Org. Chem. 2014, 34, 362 (in Chinese).

(于沙沙, 徐希森, 刘洋, 李家柱, 金英学, 祁彩霞, 王进 军, 有机化学, 2014, 34, 362.)

(b) Tamiaki, H.; Monobe, R.; Koizumi, S.; Miyatake, T.; Kinoshita, Y. Tetrahedron: Asymmerty 2013, 24, 966.

(c) Srivatsan, A.; Wang, Y.-F.; Joshi, P.; Sajjad, M.; Chen, Y.-H.; Liu, C.; Thankcppan, K.; Missert, J. R.; Tracy, E.; Morgan, J.; Rigual, N.; Baumann, H.; Pandey, R. K. J. Med. Chem. 2011, 54, 6859.

(d) Yin, Y.-F.; Zhang, Q.; Liu, Y.; Xu, X.-S.; Qi, C.-X.; Wang, J.-J. Chin. J. Org. Chem. 2013, 33, 581 (in Chinese).

(殷一㚞, 张千, 刘洋, 徐希森, 祁彩霞, 王进军, 有机化 学, 2013, 33, 581.)

[9] Wang, J.-J.; Wang, P.; Li, J.-Z.; Jakus, J.; Shin, Y.-K. Bull. Korean Chem. Soc. 2011, 32, 3473

[10] Liu, R.-R.; Wang, L.-M.; Yin, J.-G.; Wu, J.; Liu, C.; Zhang, P.; Wang, J.-J. Chin. J. Org. Chem. 2012, 32, 318 (in Chinese).

(刘舟手, 王鲁敏, 殷军港, 武进, 刘超, 张朋, 王进军, 有 机化学, 2012, 32, 318.)

[11] Liu, C. M.S. Thesis, Yantai University, Yantai, 2010 (in Chinese). (刘超, 硕士论文, 烟台大学, 烟台, 2010.)

[12] Han, G.-F.; Wang, J.-J.; Qu, Y.; Shin, Y.-K. Chin. J. Org. Chem. 2006, 26, 43 (in Chinese).

(韩光范, 王进军, 賱燕, 沈荣基, 有机化学, 2006, 26, 43.) 